\title{
The development of discontinuous Galerkin methods
}

\author{
Bernardo Cockburn $^{1}$, George E. Karniadakis ${ }^{2}$, and Chi-Wang Shu ${ }^{2}$ \\ 1 School of Mathematics, University of Minnesota, Minneapolis, Minnesota \\ 55455 , USA \\ 2 Division of Applied Mathematics, Brown University, Providence, Rhode Island \\ 02912, USA
}

\begin{abstract}
In this paper, we present an overview of the evolution of the discontinuous Galerkin methods since their introduction in 1973 by Reed and Hill, in the framework of neutron transport, until their most recent developments. We show how these methods made their way into the main stream of computational fluid dynamics and how they are quickly finding use in a wide variety of applications. We review the theoretical and algorithmic aspects of these methods as well as their applications to equations including nonlinear conservation laws, the compressible Navier-Stokes equations, and Hamilton-Jacobi-like equations.
\end{abstract}

\section{Introduction}

Problems of practical interest in which convection plays an important role arise in applications as diverse as meteorology, weather-forecasting, oceanography, gas dynamics, aeroacoustics, turbomachinery, turbulent flows, granular flows, oil recovery simulation, modeling of shallow water, transport of contaminant in porous media, viscoelastic flows, semiconductor device simulation, magneto-hydrodynamics, and electro-magnetism, among many others. This is why devising robust, accurate, and efficient methods for numerically solving these problems is of considerable importance and, as expected, has attracted the interest of many researchers and practitioners.

This endeavor, however, is far from trivial because of two main reasons. The first is that the exact solution of (nonlinear) purely convective problems develops discontinuities in finite time; the second is that these solutions might display a very rich and complicated structure near such discontinuities. Thus, when constructing numerical methods for these problems, it must be guaranteed that the discontinuities of the approximate solution are the physically relevant ones. Also, it must be ensured that the appearance of a discontinuity in the approximate solution does not induce spurious oscillations that spoil the quality of the approximation; on the other hand, while ensuring this, the method must remain sufficiently accurate near that discontinuity in order to capture the possibly rich structure of the exact solution.

These difficulties were successfully addressed during the remarkable development of the high-resolution finite difference and finite volume schemes for 
nonlinear hyperbolic systems by means of suitably defined numerical fluxes and slope limiters. Since discontinuous Galerkin (DG) methods assume discontinuous approximate solutions, they can be considered as generalizations of finite volume methods. As a consequence, the DG methods incorporate the ideas of numerical fluxes and slope limiters into the finite element framework in a very natural way; they are able to capture the physically relevant discontinuities without producing spurious oscillations near them; see an illustration of this fact in Fig. 1. Notice that the solution itself is not monotone, however the overshoot and undershoot are not significant and the averages of the solution on the elements are monotone.

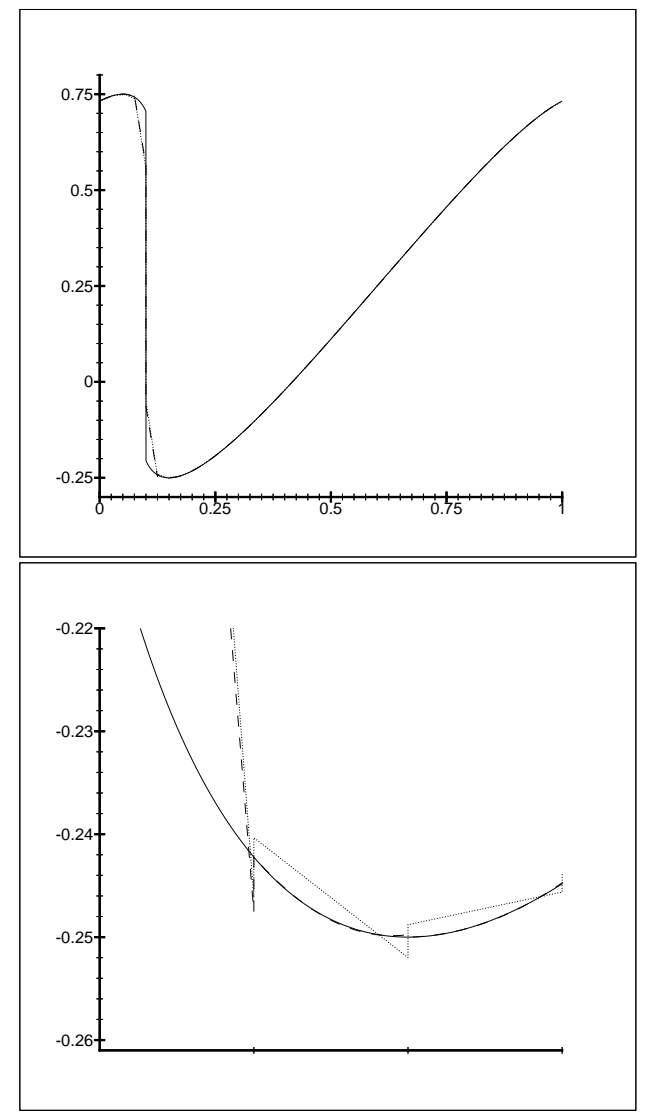

Fig. 1. Burgers equation with periodic boundary conditions and initial data $1 / 4+$ $\sin (\pi(2 x-1)) / 2$. Comparison of the exact and the approximate solutions obtained with $\Delta x=1 / 40$ at $T=0.40$. Top: full domain, bottom: detail; exact solution (solid line), piecewise linear solution (dotted line), and piecewise quadratic solution (dashed line). 
Owing to their finite element nature, the DG methods have the following main advantages over classical finite volume and finite difference methods:

- The actual order of accuracy of DG methods solely depends on the exact solution; DG methods of arbitrarily high formal order of accuracy can be obtained by suitably choosing the degree of the approximating polynomials.

- DG methods are highly parallelizable. Since the elements are discontinuous, the mass matrix is block diagonal and since the size of the blocks is equal to the number of degrees of freedom inside the corresponding elements, the blocks can be inverted by hand (or by using a symbolic manipulator) once and for all.

- DG methods are very well suited to handling complicated geometries and require an extremely simple treatment of the boundary conditions in order to achieve uniformly high-order accuracy.

- DG methods can easily handle adaptivity strategies since refinement or unrefinement of the grid can be achieved without taking into account the continuity restrictions typical of conforming finite element methods. Moreover, the degree of the approximating polynomial can be easily changed from one element to the other. Adaptivity is of particular importance in hyperbolic problems given the complexity of the structure of the discontinuities.

Although the original DG method has been known since 1973, it was only recently that DG methods have evolved in a manner that made them suitable for use in computational fluid dynamics and the aforementioned applications. In this paper, we introduce the DG methods and give an overview of their evolution since their introduction in 1973 by Reed and Hill [145], in the framework of transport of neutrons, until their most recent applications, as well as their theoretical and computational developments.

This paper is organized as follows. In section 2, we present the original DG method and describe its theoretical and computational developments in the framework of linear hyperbolic systems and ordinary differential equations. We also review other early applications, its use to discretize in time parabolic problems, and its introduction to the numerical approximation of viscoelastic flows.

In section 3, we present the evolution of the DG method for nonlinear hyperbolic problems. We show how the first attempts to extend the original DG method lead to implicit schemes and how the efforts to use explicit schemes lead to the construction of the so-called Runge-Kutta DG (RKDG) methods. We show how the RKDG methods incorporated the ideas of numerical flux and slope limiter into the finite element framework to produce formally high-order accurate, nonlinearly stable schemes. Finally, numerical applications to the Euler equations of gas dynamics are displayed.

In section 4, we review how the DG methods were extended to convectiondiffusion systems. After presenting some early attempts involving the use of 
standard mixed methods, we describe the method used by Bassi and Rebay whose generalization lead to the so-called local DG (LDG) methods. Then, we display applications to the compressible Navier-Stokes equations and to MHD. Finally, we mention the Baumann-Oden DG method for the discretization of second-order equations and several new developments.

In section 5, we describe the extension of the RKDG method to HamiltonJacobi equations and the extension of the LDG method to second-order nonlinear degenerate parabolic equations. We present an application to movement by mean curvature.

In section 6 , we briefly discuss parallelization and adaptivity for the DG methods. We also discuss several implementational issues. The first is the use of an orthogonal, tensor-product basis for unstructured grids in 2D and 3D. We also discuss quadrature-free implementations of DG methods and point out the object-oriented codes currently in use.

We end this review in section 7 , which is devoted to the discussion of open problems and future developments.

\section{Linear hyperbolic systems}

\subsection{The original DG method for the neutron transport problem}

The original DG finite element method was introduced in 1973 by Reed and Hill [145] for solving the neutron transport equation

$$
\sigma u+\nabla \cdot(\mathbf{a} u)=f, \quad \text { in } \Omega,
$$

where $\sigma$ is a real number and a a constant vector. The relevance of the method was recognized by LeSaint and Raviart who in 1974 [117] published its first mathematical analysis.

To display the method, we multiply the equation by a test function $v$ and integrate over an arbitrary subset of $\Omega$, say $K$. After a formal integration by parts, we get

$$
\sigma(u, v)_{K}-(u, \mathbf{a} \cdot \nabla v)_{K}+\left\langle\mathbf{a} \cdot \mathbf{n}_{K} u, v\right\rangle_{\partial K}=(f, v)_{K},
$$

where $\mathbf{n}_{K}$ denotes the outward unit normal of $\partial K$, and

$$
(u, v)_{K}=\int_{K} u v d x, \quad\langle w, v\rangle_{\partial K}=\int_{\partial K} w v d s .
$$

Next, we construct a triangulation $\mathcal{T}_{h}=\{K\}$ of $\Omega$, and take our approximate solution $u_{h}$ to be a polynomial of degree at most $k$ on each element $K$ of the triangulation. The approximate solution $u_{h}$ is then determined as the unique solution of the following weak formulation:

$$
\begin{aligned}
& \forall K \in \mathcal{T}_{h}: \\
& \sigma\left(u_{h}, v\right)_{K}-\left(u_{h}, \mathbf{a} \cdot \nabla v\right)_{K}+\langle\hat{h}, v\rangle_{\partial K}=(f, v)_{K}, \quad \forall v \in P^{k}(K),
\end{aligned}
$$


where $P^{k}(K)$ denotes the space of polynomials of degree at most $k$ on the element $K$ and $\hat{h}$ is the numerical flux given by

$$
\hat{h}(\mathbf{x})=\mathbf{a} \cdot \mathbf{n}_{K}(x) \lim _{s \downarrow 0} u_{h}(\mathbf{x}-s \mathbf{a}) .
$$

Note that the value $\lim _{s \downarrow 0} u_{h}(\mathbf{x}-s \mathbf{a})$ is nothing but the value of $u_{h}$ upstream the characteristic direction a. As a consequence, the degrees of freedom of the approximate solution $u_{h}$ in the element $K$ can be computed in terms of the values of $u_{h}$ upstream the characteristics hitting $\partial K$. In other words, the approximate solution $u_{h}$ can be computed element by element when the elements are suitably ordered according to the characteristic direction $\mathbf{a}$.

\subsection{The DG method for ODEs}

The first analysis of the DG method as applied to ODEs, was performed in 1974 by LeSaint and Raviart [117] who showed that the method is strongly Astable of order $2 k+1$ at mesh points, and that the Gauss-Radau discretization of the DG method is also of order $2 k+1$ when piecewise polynomials of degree $k$ are used.

It is interesting to note that only one year before the introduction of the DG method by Reed and Hill, Hulme $[107,108]$ had studied a method for ODEs which used the same weak formulation as the DG method but employed a continuous approximate solution $u_{h}$; this method is, however, only of order $2 k$ at mesh points. A study of global error control for ODEs for this method was carried out in 1994 by Estep and French [84]. Another very interesting work on DG methods for ODEs was done in 1981 by Delfour, Hager and Trochu [70]; they introduce a class of DG methods which are proven to give an order of accuracy up to $2 k+2$ at the mesh points. Recently, Schötzau and Schwab have obtained a new estimate on the size of the time step needed to solve the implicit system of equations determined by the DG method by means of a simple fixed point iteration technique; see the reference in the lecture notes by Schwab [152].

In 1988, Johnson [112] gave an analysis of error control for the DG method for stiff ODEs and later in 1995, Estep [83] extended this analysis to general non-autonomous ODEs. Finally, in 1996, Böttcher and Rannacher [37]

introduced a new adaptive error control technique for ODEs by using the DG method.

\subsection{Analysis of the original DG method}

A priori error estimates. In 1974, LeSaint and Raviart [117] made the first analysis of the DG method and proved a rate of convergence of $(\Delta x)^{k}$ in the $\mathrm{L}^{2}(\Omega)$-norm for general triangulations and of $(\Delta x)^{k+1}$ for tensor products of polynomials of degree $k$ in one variable defined on Cartesian grids. In 1986, Johnson and Pitkaränta [113] proved a rate of convergence of $(\Delta x)^{k+1 / 2}$ for 
general triangulations and in 1991, Peterson [140] numerically confirmed this rate to be optimal. In 1988, Richter [146] obtained the optimal rate of convergence of $(\Delta x)^{k+1}$ for some structured two-dimensional non-Cartesian grids. The issue of the loss of order of convergence was addressed again in 1991 by Lin and Zhou [120] who proved that the standard Galerkin method using bilinear approximations defined on almost uniform Cartesian is of order 2; the order of this method for arbitrary meshes is only one. In 1994, Zhou and Lin [180] extended this result to piecewise-linear approximations in almost uniform triangulations. Then, in 1996 Lin, Yan, and Zhou [119] showed first order convergence for the DG method using piecewise-constant approximations. Their result holds for almost uniform grids of rectangles and for almost uniform grids of triangles; their technique is based on a key approximation result. In this volume, Lin [118] reviews this technique and applies it to several finite element approximations for hyperbolic problems. Also in this volume, Falk [86] reviews several techniques of analysis for finite element methods for hyperbolic problems including the DG method and the continuous Galerkin method.

All the above mentioned papers assume that the exact solution is smooth. In 1993, Lin and Zhou [121] proved convergence to the weak solution assuming only that the exact solution belongs to $H^{1 / 2}(\Omega)$. More recently, Houston, Schwab and Süli [102] proved spectral convergence of the DG method assuming that the exact solution is piecewise analytic. In this volume, E. Süli, Ch. Schwab, and P. Houston [162] review these results and extends them to $h p$ DGFEM for PDEs with non-negative characteristic form. Finally, Cockburn, Luskin, Shu and Süli [54] showed that if the exact solution is in $\mathrm{L}^{2}$ but is locally smoother, error estimates can be obtained between the exact solution and a suitably post-processed approximate solution.

Concerning the issue of super-convergence, in 1994, Biswas, Devine and Flaherty [36] discovered that the approximate solution of the DG method super-converges at the Gauss-Radau points. A rigorous proof of this fact was recently found by Adjerid, Flaherty, and Krivodonova [3]; the groundwork for this analysis was carried out in 1998 by Adjerid, Aiffa and Flaherty [1]. Another indication of super-convergence was obtained by Lowrie [128] who reported numerical evidence of the existence of a component of the error of the DG method that was $(2 k+1)$-th. order accurate. This experimental indication was put on firm mathematical basis by Cockburn, Luskin, Shu and Süli [55] who showed that, assuming that the exact solution is sufficiently smooth, a simple post-processing of the approximate solution obtained with polynomials of degree $k$ does produce an approximation of order $2 k+1$; in this volume, they present a short version of this result. Also in this volume, Lin [118] proposes a new error estimation technique for finite element approximations of hyperbolic problems.

A posteriori error analysis. In 1990, Stroubolis and Oden [158] studied a posteriori error estimates for the DG method. Later, Bey and Oden [33] 
obtained the first $h p$ - a posteriori error estimates for the DG method; parallelization strategies based on these estimates were developed in 1995 by Bey, Patra, and Oden [35] and in 1996 by Bey, Oden and Patra [34].

A posteriori error analysis of finite element methods for hyperbolic problems, including a slight modification of the original DG method, have been studied in 1996 by Süli [159] and in 1997 by Süli and Houston [161]; see also the 1999 lectures notes on this subject by Süli [160].

Wave propagation analysis. An analysis of wave propagation for the DG method is given in this volume by Rasetarinera, Hussaini, and $\mathrm{Hu}$ [143]. Also in this volume is a paper by Sherwin [154] which is devoted to the study of numerical phase properties of continuous and discontinuous Galerkin methods using a high-order basis (see section 6.2).

\subsection{Early applications of the DG method}

Besides the application of the DG to the simulations of neutron transport and to ODEs, applications of this method to the analysis of wave propagation in elastic media was done from 1975 to 1976 by Oden and Wellford $[173,134,174,175]$, and to optimal control in 1978 by Delfour and Trochu [71].

\subsection{Time discretization of parabolic equations}

Also in 1978, Jamet [110] used the DG method to discretize in time parabolic equations and showed that the method was of order $k$. Since then, several authors have studied this method. Thus, in 1985, K. Eriksson, C. Johnson and V. Thomée [82] proved that the method was of order $2 k+1$ at the nodes and later Erikson and Johnson studied the issue of error control in a series of papers [77-81] starting in 1987 and ending in 1995. In 1997, Makridakis and Babuška [132] studied the effect on adaptive mechanisms on the stability of the method. In this volume, Machiels [130] investigates an adaptive procedure for this method based on a new a posteriori error control. Also in this volume, Estep and Freund [85] use it to solve nonlinear reaction-diffusion systems; they show how to use an inexact Newton method preconditioned with Krylov-subspace iteration. Finally, Schötzau and Schwab have studied how to actually solve the system of equations defined by the DG methods; they show that it is possible to decouple the system into several scalar equations of the same type; see the lecture notes by Schwab [152].

\subsection{DG methods for viscoelastic flows}

In 1989, the DG method of Reed and Hill was applied for the first time for the numerical computation of viscoelastic flows by Fortin and Fortin [93]. The idea was to apply the DG method to the constitutive law relating the 
so-called extra-stress tensor in terms of the velocity. In this volume, Fortin, Béliveau, Heuzey and Lioret [92] review the development of this idea and Baaijiens, Bogaerds and Verbeeten [13] study the successes and failures of the use of these methods in viscoelastic fluid analysis. A recent application of the DG to these problems was pursued in 1998 by Sun, Smith, Armstrong, and Brown [163].

Mathematical analysis of these methods have been carried out in 1992 by Baranger and Sandri [21], in 1995 by Baranger and Wardi [22], in 1997 by Baranger and Machmoum [20], and in 1998 by Bahhar, Baranger and Sandri [14]. See also the 1996 paper by Baranger and Machmoum [19].

\subsection{New developments: DG methods for Maxwell's equations}

The equations of (viscous) magneto-hydrodynamics, that include the Maxwell's equations, have been discretized with DG methods by Warburton and Karniadakis [171]. Other applications to the Maxwell's equations are presented in three papers in this volume. Warburton [169] presents the use of the DG method with unstructured polymorphic $h p$-finite elements; Kopriva, Woodruff and Hussaini [116] consider a spectral discontinuous method; and Cai [39] deals with the problem of defining the basis functions for electromagnetic scattering of curved surfaces.

\section{Nonlinear hyperbolic systems}

\subsection{The space DG-discretization}

The success of the DG method for linear hyperbolic problems, made the extension to the nonlinear hyperbolic systems

$$
\mathbf{u}_{t}+\sum_{i=1}^{d}\left(\mathbf{f}_{i}(\mathbf{u})\right)_{x_{i}}=0
$$

the natural step in the development of the method. An extension of the original DG method can be obtained as follows. To simplify the presentation, let us assume that $u$ is a scalar-valued function; in the case of a vector-valued $\mathbf{u}$, we proceed similarly component by component. Thus, we multiply the above equation by a test function and formally integrate by parts to get

$$
\left(u_{t}, v\right)_{K}-\sum_{i=1}^{d}\left(\mathbf{f}_{i}(u), \partial_{x_{i}} v\right)_{K}+\sum_{i=1}^{d}\left\langle\mathbf{f}_{i}(u)\left(\mathbf{n}_{K}\right)_{i}, v\right\rangle_{\partial K}=0 .
$$

The approximate solution $u_{h}$ is now defined as the solution of the following weak formulation:

$$
\begin{aligned}
& \forall K \in \mathcal{T}_{h}: \\
& \left(\left(u_{h}\right)_{t}, v\right)_{K}-\sum_{i=1}^{d}\left(\mathbf{f}_{i}\left(u_{h}\right), \partial_{x_{i}} v\right)_{K}+\langle\hat{h}, v\rangle_{\partial K}=0, \quad \forall v \in P^{k}(K),
\end{aligned}
$$


where $\hat{h}$ is an approximation to the trace of $\sum_{i=1}^{d} \mathbf{f}_{i}(u)\left(\mathbf{n}_{K}\right)_{i}$ on the boundary of the element $K$, in other workds, it is nothing but an approximate Riemann solver; see, for example, Toro [166] and the references therein. This shows that the treatment of the boundary conditions is natural and extremely simple. Chavent and Salzano [44] used the above DG-space discretization in 1982 for the first time in the framework of nonlinear conservation laws.

Now, it only remains to discretize the above equations in time. However, it is not simple to find a time discretization that would result in a stable, efficient, and formally high-order accurate method. At this point in the development of the DG methods for hyperbolic conservation laws, this was the main difficulty.

\subsection{Implicit time-discretizations}

Global time-discretizations. The presence of the nonlinearities $\mathbf{f}_{i}$ prevents the element-by-element computation of the solution that was possible in the linear case considered by Reed and Hill [145]. This is so because it is no longer possible to determine the characteristics explicitly. Thus, one is forced to use implicit time discretizations and hence, to solve at each time step a new nonlinear system of equations. This renders the method computationally very inefficient for hyperbolic problems. In 1989, Bar-Yoseph [17] and in 1990, Bar-Yoseph and Elata [18] explored this approach.

Local time-discretizations. A way around this difficulty was found independently in 1994 by Richter [148], in 1996 by Lowrie [128] and Lowrie, Roe and, van Leer [126], [127] and the work in [157], [179], and [40] by the group of Haber and his collaborators. It consists of using space-time elements constructed in such a way that a local element-by-element computation is still possible. In this volume, Lowrie and Morel [129] use this approach to deal with hyperbolic systems with stiff relaxation; Carranza, Fang, and Haber [40] use a space-time DM method to the simulation of oxidation-driven fractures in super-alloys; and Yin, Acharya, Sobh, Haber, and Tortorelli [179] apply this technique to perform elastodynamic analysis (see the application to precipitate nucleation and growth in aluminum alloy quench processes by Sobh, Huang, Yin, Haber, and Tortorelli [157]). Also in this volume, Richter [149] considers several ways to carry out this approach.

It is interesting to note that in 1990, Hulbert and Hughes [106] proposed a space-time finite element method for elastodynamics that used a DG method in time and a continuous-in-space approximation. This work, reminiscent of the approach took in 1978 by Jamet [110] for parabolic equations, can now be seen as a step toward the development of fully space-time DG methods for hyperbolic problems.

Analysis of the DG method. To rigorously analyze the DG method in the nonlinear case is very difficult; in fact, up to date there are only three results 
in this direction. The first was established in 1994 by Jiang and Shu [111] for one-dimensional nonlinear conservation laws with strictly convex or concave nonlinearities. It states that if the approximate solution converges, it converges to the entropy solution; this holds for any degree of the approximating polynomials.

The other two results hold for a version of the space-time DG method for scalar nonlinear conservation laws that contains an additional term called the shock-capturing term. In 1995, Jaffré, Johnson, and Szepessy [109] proved the convergence of the approximate solution to the entropy solution. In 1996, Cockburn and Gremaud [50] obtained the only a posteriori error estimate for this method in the nonlinear case; they also proved, not only convergence, but also an error estimate that yields the order of convergence of $1 / 4$ in $L^{\infty}\left(0, T ; L^{1}\right)$ for possibly discontinuous solutions. These results hold in any number of space dimensions and for any value of the polynomial degree.

\subsection{Explicit schemes: The Runge-Kutta Discontinuous Galerkin methods}

The Euler method. To avoid the difficulty of implicit time discretizations, in 1982, Chavent and Salzano [44] constructed an explicit version of the DG method in the case of a one-dimensional scalar conservation law. They discretized in space by using the DG method with piecewise linear elements and then discretized in time by using the simple forward Euler method. Unfortunately, a classical von Neumann analysis shows that the resulting method is unconditionally unstable when the ratio $\frac{\Delta t}{\Delta x}$ is held constant; it is stable if $\frac{\Delta t}{\Delta x}$ is of order $\sqrt{\Delta x}$. This condition is reasonable if the method is used in conjunction with explicit methods for convection-diffusion schemes, as done for secondary oil recovery by Chavent and Jaffré [43], but it is a very restrictive condition for hyperbolic problems.

Incorporation of the slope limiter. To improve the stability of the scheme, in 1989, Chavent and Cockburn [42] modified the scheme by introducing a suitably defined slope limiter, following the ideas introduced in 1974 by van Leer [168]. They thus obtained a scheme that was proven to be total variation diminishing in the means (TVDM) and total variation bounded (TVB) provided that the CFL number, $f^{\prime} \frac{\Delta t}{\Delta x}$, is less than or equal to $1 / 2$; convergence of a subsequence is thus guaranteed. Although the numerical results indicate convergence to the correct entropy solutions, the scheme is only first order accurate in time. Moreover, the slope limiter has to balance the spurious oscillations in smooth regions caused by linear instability, hence adversely affecting the quality of the approximation in these regions.

The first RKDG method. These difficulties were overcome by Cockburn and Shu in [58], where the first Runge Kutta Discontinuous Galerkin (RKDG) 
method was introduced. This method was constructed by (i) retaining the piecewise linear DG method for the space discretization, (ii) using a special explicit TVD second order Runge-Kutta type discretization introduced by Shu and Osher in 1988 [155] and in 1989 [156], and (iii) modifying the slope limiter to maintain the formal accuracy of the scheme at extrema. The resulting explicit scheme was then proven to be linearly stable for CFL numbers less than $1 / 3$, formally uniformly second order accurate in space and time, and total variation bounded in the means (TVBM). The numerical results show second order convergence in smooth regions including at extrema, sharp shock transitions (usually in one or two elements) without oscillations, and convergence to entropy solutions even for non convex fluxes.

High-order accurate RKDG methods. In 1989, Cockburn and Shu [56] generalized this approach and constructed (formally) high-order accurate RKDG methods for the scalar hyperbolic conservation law. To device RKDG methods of order $k+1$, they used (i) the DG-space discretization method with polynomials of degree $k$ for the space discretization, (ii) a TVD Runge-Kutta $(k+1)$-th order accurate explicit time discretization, and (iii) a generalized slope limiter. The generalized slope limiter was carefully devised to enforce the TVBM property without destroying the accuracy of the scheme. The numerical results, for $k=1,2$, indicate $(k+1)$-th order order in smooth regions away from discontinuities as well as sharp shock transitions with no oscillations; convergence to the entropy solutions was observed in all the tests.

In 1994, Biswas, Devine, and Flaherty [36] introduced a new generalized slope limiter. Although no stability results have been proven for this generalized slope limiter, it has the advantage of dealing with local critical points without the aid of any auxiliary parameter. Another distinctive feature is that it can be readily used for $h p$-adaptivity purposes.

One-dimensional systems. These RKDG schemes were extended to onedimensional systems in 1989 by Cockburn, Lin and Shu [53].

Multi-dimensional scalar equations. The extension of the RKDG method to the scalar multi-dimensional case was done in 1990 by Cockburn, Hou, and Shu [51]. The main contributions of this extension are (i) some accuracy considerations, and (ii) the extension of the generalized slope limiter.

It was found that in order to ensure formal accuracy of order $k+1$ when using polynomials of degree $k$, quadrature rules, exact for polynomials of degree $2 k$, should be used for the integrals inside the elements and quadrature rules, exact for polynomials of degree $2 k+1$, should be used for the integrals on the faces of the elements.

The construction of the generalized slope limiter was not simple. This is so, not only because of the more complicated form of the elements but 
also because of inherent accuracy barriers imposed by the stability properties. Indeed, since the main purpose of the slope limiter is to enforce the nonlinear stability of the scheme, it is essential to realize that in the multidimensional case the constraints imposed by the stability of a scheme on its accuracy are even greater than in the one-dimensional case. Although in the one-dimensional case it is possible to devise high-order accurate schemes with the TVD property, this is not true in several space dimensions, since in 1985, Goodman and LeVeque [96] proved that any TVD scheme is at most first order accurate. Thus, any generalized slope limiter that enforces the TVD property, or the TVDM property for that matter, would unavoidably reduce the accuracy of the scheme to first order accuracy. This is why Cockburn, Hou and Shu [51] devised a generalized slope limiter that enforced a local maximum principle only; maximum principles are not incompatible with high-order accuracy. No other class of schemes of second or higher order of accuracy has a proven maximum principle for general nonlinearities $\mathbf{f}$ and unstructured triangulations.

In 1997, Wierse [177] introduced and studied several interesting new slope limiters for formally high-order accurate schemes defined in unstructured triangulations.

Multi-dimensional systems. The extension of the RKDG methods to general multi-dimensional systems was initiated in 1991 by Cockburn and Shu in [57] and was completed in 1998 in [60] where applications to the Euler equations of gas dynamics were displayed. One of the contributions of [60] is the construction of a new, practical generalized slope limiter which works very well in triangles and rectangles and with piecewise linear and quadratic elements.

In 1996, Devine and Flaherty [73] introduced a parallel adaptive $h p$ refinement techniques for conservation laws using the RKDG methods.

Numerical experiments for the Euler equations of gas dynamics were performed in 1991 by Bey and Oden [32], in 1997 by Bassi and Rebay [26], in 1998 by Baumann and Oden [31] and by Warburton, Lomtev, Kirby, and Karniadakis [172].

\subsection{Other explicit time-stepping schemes}

Time-stepping schemes different from the TVD Runge-Kutta time-stepping used by the RKDG can give very good computational results. However, it remains to be proven that those methods share with the RKDG methods the same nonlinear stability properties.

\subsection{The DG methods of Allmaras and Halt}

Totally independently of the just described development of DG methods, Allmaras and then Halt explored schemes which would now be considered 
DG methods. In 1989, Allmaras [5] introduced a DG method for the Euler equations of gas dynamics; an earlier version of his algorithm appeared in the 1987 paper by Allmaras and Giles [6]. He used the Roe parametric variables, piecewise linear test functions, a two-point Gauss quadrature rule on the edges, and a three-stage second-order Runge-Kutta time stepping method; he also took into account the curvature of the boundaries of the domain. In 1992, Halt [99] extended Allmaras' work to higher degree polynomials and to general unstructured grids in two- and three-space dimensions. His numerical test cases include the Ringleb flow, 2-D airfoils and the 3-D Onera M6 wing. See also the 1991 and 1992 papers by Halt and Agarwall [100] and [101], respectively. No slope limiters were considered by the above mentioned authors.

\subsection{Numerical experiments: Gas Dynamics}

In what follows, we present some numerical results from some of the papers mentioned above and some new results that display the performance of the method when applied to the Euler equations of gas dynamics.

Approximation of the boundaries. In 1997, Bassi and Rebay [26] showed with a remarkable experiment, the importance of using a good approximation of the boundaries of the space domain. Here, we reproduce some of their results to illustrate that point.

The test problem is the classical two-dimensional isentropic flow around a circle. In Fig. 2, part of the grid is displayed and the corresponding solution using $\mathrm{P}^{1}$ elements is shown. Note that in this grid, the circle is approximated by a polygon; since each of the kinks of the polygon introduces non-physical entropy production, the approximate solution presents a non-physical wake which does not disappear by further refining the grid! By simply taking into account the exact shape of the boundary, a remarkably improved approximation is obtained, as can be seen in Fig. 3. Note also the improvement of the approximation as the degree of the polynomials is increased from 1 to 3 !

Spectral convergence. We consider the isentropic flow in the geometry shown in Fig. 4; the numerical results we show are from the work of Warburton, Lomtev, Kirby, and Karniadakis [172]. Low-order methods erroneously produce entropy from inlet to outlet for this problem. In Fig. 4 (bottom), we show that the entropy errors converge exponentially fast to zero as the degree of the polynomials increases. A comparison is shown on the plot of the bottom between a fully unstructured and a hybrid discretization; more elements are used in the unstructured grid.

Approximation of contact discontinuities. Now, we consider the classical double-Mach reflection problem; we show results from the work of Cockburn and Shu [60]. In Fig. 5, we show details of the approximation of the 


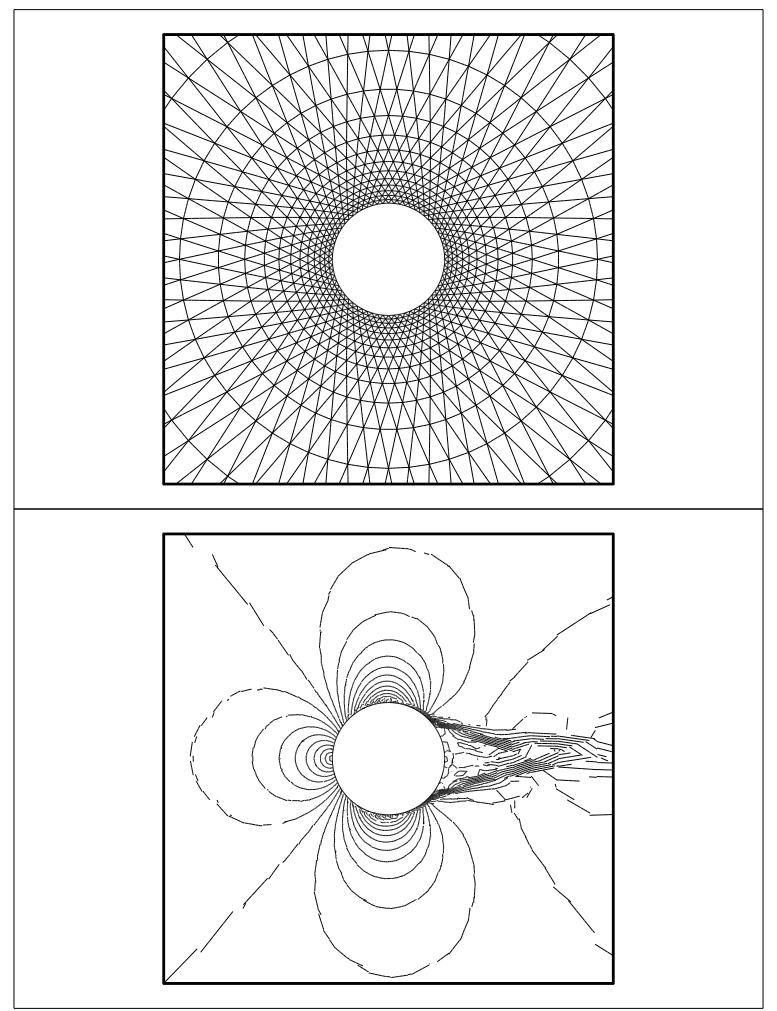

Fig. 2. Grid " $64 \times 16$ " with a piecewise linear approximation of the circle (top) and the corresponding solution (Mach isolines) using $\mathrm{P}^{1}$ elements (bottom). 


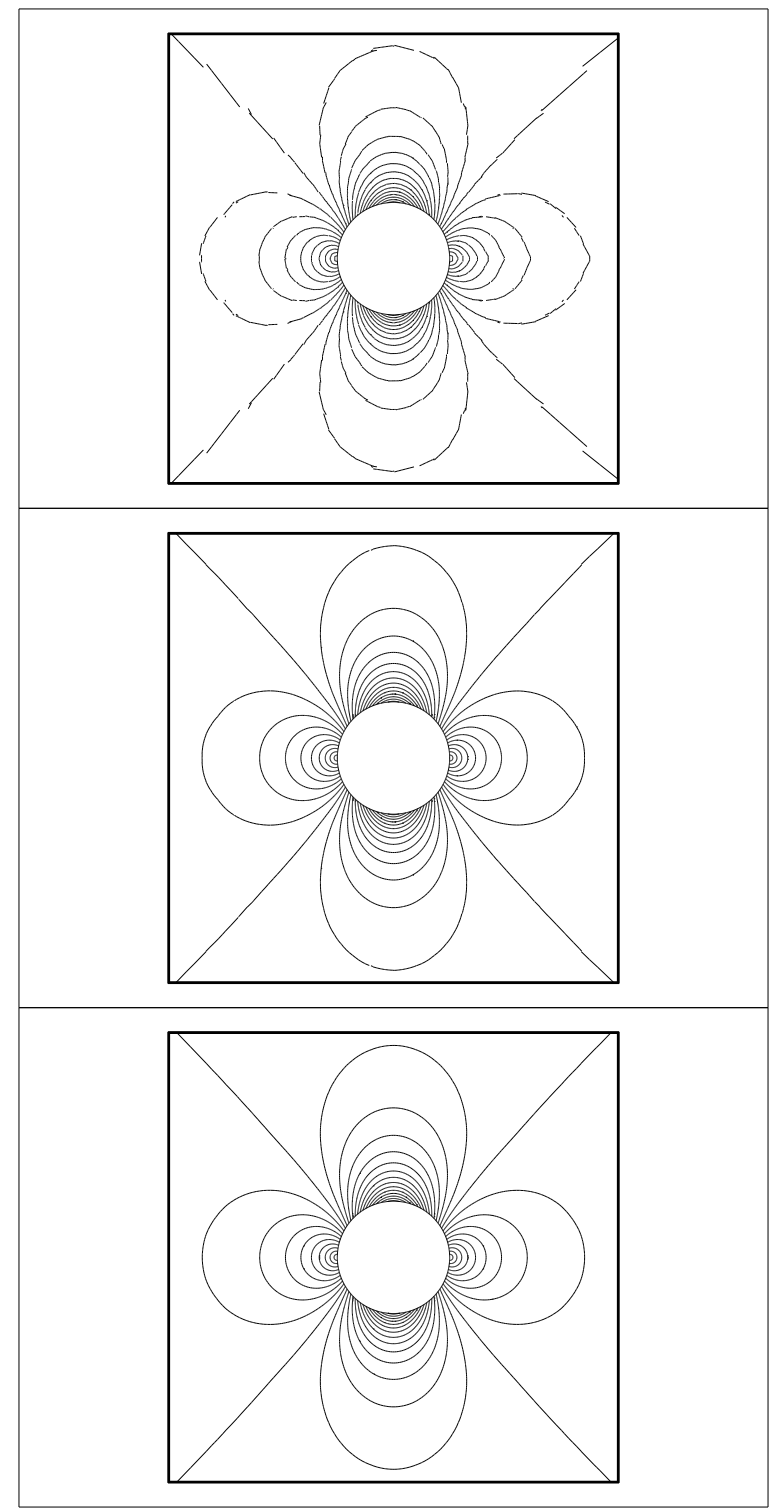

Fig. 3. Grid " $64 \times 16$ " with exact rendering of the circle and the corresponding $\mathrm{P}^{1}$ (top), $\mathrm{P}^{2}$ (middle), and $\mathrm{P}^{3}$ (bottom) approximations (Mach isolines). 

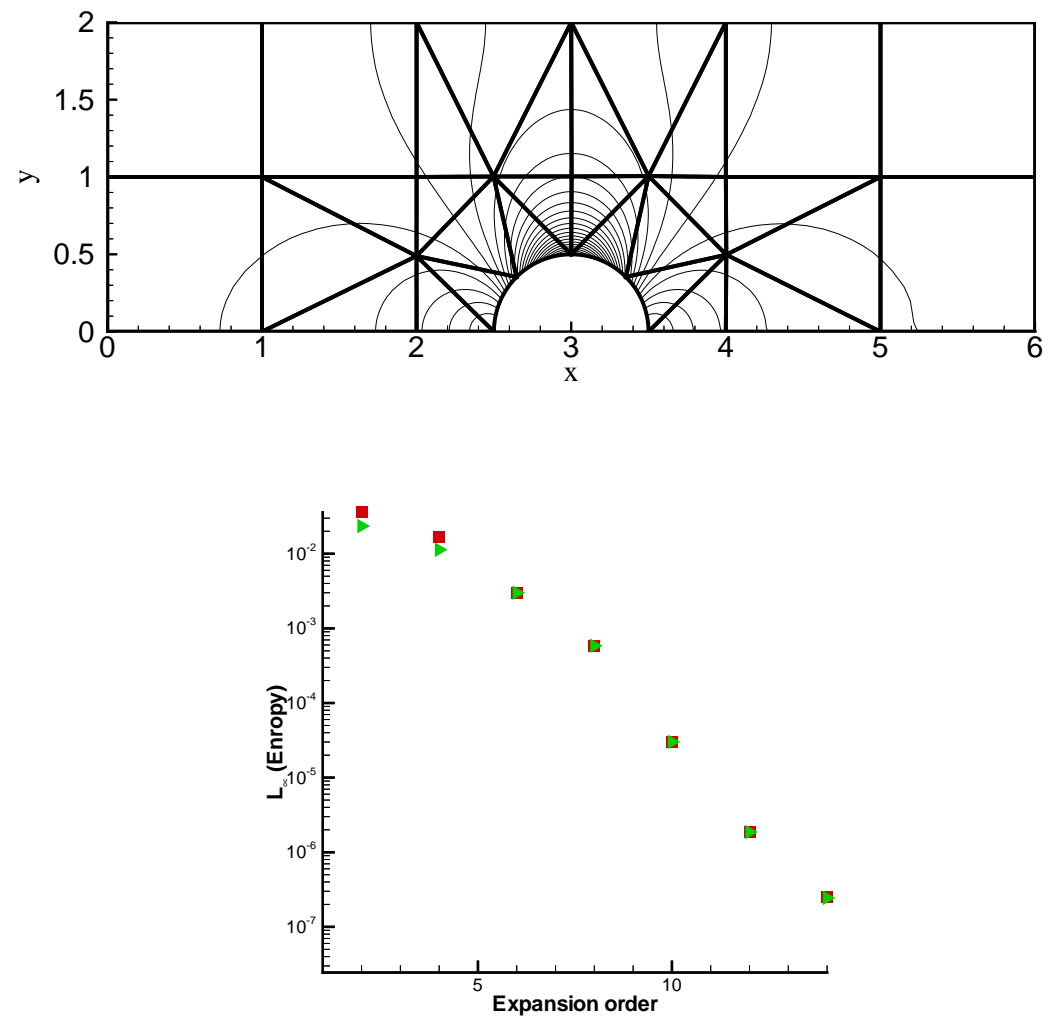

Fig. 4. Density contours (top) obtained on a hybrid grid for an inviscid $M=0.3$ flow (left). History of convergence (bottom): Exponential convergence of the error is obtained for an unstructured (triangles) and a hybrid (squares) grid. 
density. Note that the strong shocks are very well resolved with both $P^{1}$ and $P^{2}$ elements. Also, note that there is a remarkable improvement in the approximation of the density near the contacts when going from $P^{1}$ elements to $P^{2}$ elements.

Conclusions. The first experiment illustrates the fact that very good approximations of the boundaries are crucial. The second experiment shows that the DG method can achieve spectral accuracy, and so, that polynomials of high degree should be used when dealing with a smooth solution. Finally, the last experiment shows that this is also desirable even when the solution is not smooth.

\subsection{New developments and applications}

In this volume, Barth [23] presents a new simplified version of the DG methods for conservation laws that incorporates a symmetrization technique; Despres [72] presents a DG method for solving the Euler equation in an axisymmetric geometry; Gremaud [98] presents an application of the DG method to granular flow; and, van der Ven and van der Vegt [167] present a study of the accuracy, resolution and computational complexity of a DG method.

\section{Convection-diffusion systems}

\subsection{A DG method for convection-diffusion problems}

In 1992, Richter [147] proposed a direct extension of the original DG method to linear convection-diffusion equations. Richter proved that if the convection is dominant, that is, if the viscosity coefficients were of the order of the meshsize, the optimal order of convergence is $k+1 / 2$ when polynomials of degree $k$ are used.

\subsection{A coupled Euler/Navier-Stokes solver}

In 1989, Allmaras [5], see also Allmaras and Giles [7], proposed to couple his DG method for the Euler equations of gas dynamics and a compressible Navier-Stokes solver. The two solvers were applied to different, overlapping regions of the computational domain.

\subsection{The Upwind-mixed methods for advection-diffusion equations}

In 1991, Dawson [63] introduced the so-called upwind-mixed methods (UMM) for advection-diffusion problems. The main idea of these methods is to combine a mixed finite element approximation for the second-order terms with an 

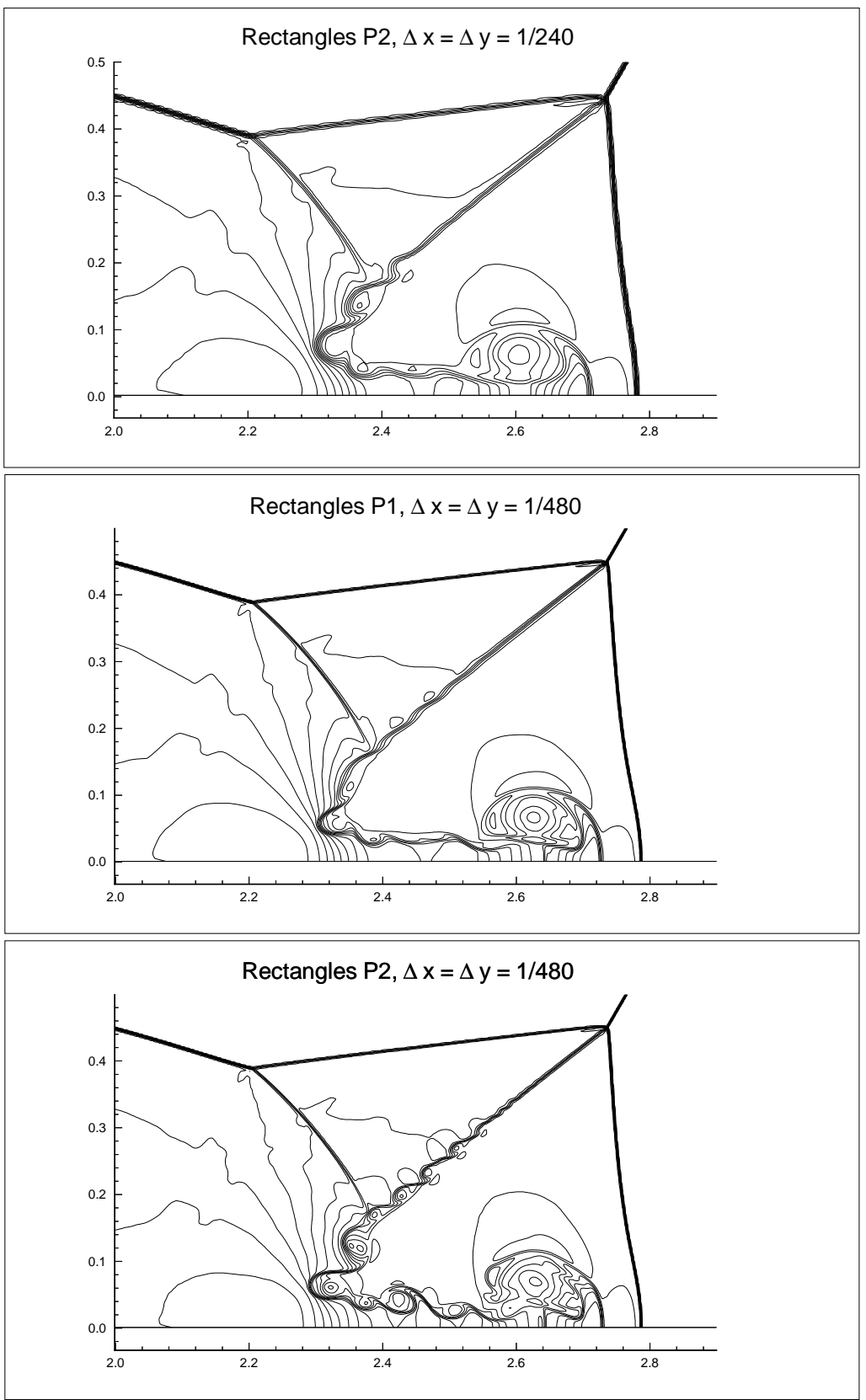

Fig. 5. Double Mach reflection problem. Blown-up region around the double Mach stems. Density $\rho$. Third order $P^{2}$ with $\Delta x=\Delta y=\frac{1}{240}$ (top); second order $P^{1}$ with $\Delta x=\Delta y=\frac{1}{480}$ (middle); and third order $P^{2}$ with $\Delta x=\Delta y=\frac{1}{480}$ (bottom). 
upwinding for the advective terms. Since the UUM always use discontinuous approximations for the solution, this is a very natural combination. In 1993, Dawson [64] extended his analysis to multi-dimensions, and in 1998, [66], analyzed the application of the method to nonlinear contaminant transport equations. All this work was done for the lowest-order Raviart-Thomas space. Recently, Dawson and Aizinger [67] considered the UMM that uses the DG method as its so-called upwinding scheme and obtained error estimates for arbitrary degree polynomial spaces. For applications of UMM to transport problems arising in porous media, see the references in [67].

\subsection{A DG method for semiconductor device simulation}

Strongly related with the above UMM method are the extensions of the RKDG method to nonlinear, convection-diffusion systems of the form

$$
\partial_{t} \mathbf{u}+\nabla \cdot \mathbf{F}(\mathbf{u}, D \mathbf{u})=0, \text { in }(0, T) \times \Omega,
$$

proposed in 1995 by Chen, Cockburn, Jerome, and Shu [46] for the hydrodynamic model for semiconductor device simulation and by Chen, Cockburn, Gardner, and Jerome [45] for the quantum hydrodynamic model for semiconductor device simulation. In these extensions, approximations of second and third-order derivatives of the discontinuous approximate solution were obtained by using simple projections into suitable finite elements spaces. This projection requires the inversion of global mass matrices, which in [46] and [45] are 'lumped' in order to maintain the high parallelizability of the method. Since in [46] and [45] polynomials of degree one are used, the 'mass lumping' is justified; however, if polynomials of higher degree were used, the 'mass lumping' needed to enforce the full parallelizability of the method could cause a degradation of the formal order of accuracy.

In this volume, Cockburn, Jerome, and Shu [52] review some of the above results and addresses the issue of the utility of modeling and simulation in determinining properties of semiconductors.

\subsection{DG-mixed methods for Compressible Navier Stokes}

In 1998, Lomtev, Quillen and Karniadakis [125] used the DG-space discretization method to deal with the convective part of the compressible NavierStokes equations and used a mixed method to approximate the diffusive part of the equations.

\subsection{The method of Bassi and Rebay and the LDG method}

In 1997, Bassi and Rebay [25] proposed an extension of the DG-space discretization method for the compressible Navier-Stokes equations. In this approach, the original idea of the DG-space discretization method is applied to 
both $u$ and $D u$ which are now considered as independent unknowns. Like the RKDG methods, the resulting methods are highly parallelizable methods of high-order accuracy which are very efficient for time-dependent, convectiondominated flows. In 1998, Cockburn and Shu [59] introduced the local discontinuous Galerkin (LDG) methods, which are a generalization of Bassi and Rebay's approach, and proved stability and error estimates for the method.

The basic idea to construct the LDG methods is to suitably rewrite the original system as a larger, degenerate, first-order system and then discretize it in space by the DG method. By a careful choice of this rewriting and of the numerical fluxes, nonlinear stability can be achieved even without slope limiters, just as for the RKDG method in the purely hyperbolic case; see Jiang and Shu [111]. The resulting method is element-wise conservative, a property which is particularly difficult to preserve with high-order finite elements.

The large amount of degrees of freedom and the restrictive conditions of the size of the time step for explicit time-discretizations, render the LDG methods inefficient for diffusion-dominated problems; in this situation, the use of methods with continuous-in-space approximate solutions is recommended. However, as for the successful RKDG methods for purely hyperbolic problems, the extremely local domain of dependency of the LDG methods allows a very efficient parallelization that by far compensates for the extra amount of degrees of freedom in the case of convection-dominated flows.

\subsection{The LDG method for purely diffusive problems}

The parabolic case. Next, we illustrate the definition of the LDG method as applied to the heat equation with variable diffusion coefficient $\nu(\mathbf{x})$ :

$$
u_{t}-\nabla \cdot(\nu \nabla u)=f, \quad \text { in }(0, T) \times \Omega,
$$

We then rewrite the above equation as the following first-order degenerate system:

$$
\begin{array}{ll}
u_{t}+\nabla \cdot \mathbf{q}=f, & \text { in }(0, T) \times \Omega, \\
\mathbf{q}+\nu \nabla u=0, & \text { in }(0, T) \times \Omega .
\end{array}
$$

and after multiplying by test functions $w$ and $\mathbf{v}$ and formally integrating by parts, we obtain

$$
\begin{aligned}
& \left(u_{t}, w\right)_{K}-(\mathbf{q}, \nabla w)_{K}+\langle\mathbf{q} \cdot \mathbf{n}, w\rangle_{\partial K}=(f, w)_{K} \\
& \left(\frac{1}{\nu} \mathbf{q}, \mathbf{v}\right)_{K}-(u, \nabla \cdot \mathbf{v})_{K}+\left\langle u, \mathbf{v} \cdot \mathbf{n}_{K}\right\rangle_{\partial K}=0 .
\end{aligned}
$$

We are now ready to define the LDG-space discretization method:

$$
\begin{aligned}
& \forall K \in \mathcal{T}_{h}: \\
& \left(\left(u_{h}\right)_{t}, w\right)_{K}-(\mathbf{q}, \nabla w)_{K}+\langle\hat{\mathbf{h}}, w\rangle_{\partial K}=(f, w)_{K}, \quad \forall w \in P^{k}(K), \\
& \left(\frac{1}{\nu} \mathbf{q}_{h}, \mathbf{v}\right)_{K}-\left(u_{h}, \nabla \cdot \mathbf{v}\right)_{K}+\langle\hat{u}, \mathbf{v} \cdot \mathbf{n}\rangle_{\partial K}=0, \quad \forall \mathbf{v} \in\left(P^{k}(K)\right)^{d},
\end{aligned}
$$


where $\hat{\mathbf{q}}$ and $\hat{u}$ are numerical fluxes that must be carefully defined.

Independently, in 1994 Giannakouros [95] and in 1997 Bassi and Rebay [25], took their numerical fluxes $\hat{\mathbf{q}}$ and $\hat{u}$ to be the arithmetic average of the two values of $\mathbf{q}_{h}$ and $u_{h}$ at the boundary of the elements. Bassi and Rebay [25] reported an order of convergence of order $k+1$ for even values of the polynomial degree $k$ and of order $k$ for odd values and in 1998, Cockburn and Shu [59] proved this result. In 1999, Lomtev and Karniadakis [123], showed numerical evidence that the method is exponentially accurate even for highly distorted grids.

In 1998, Cockburn and Shu [59] showed that for a fairly general class of numerical fluxes, the LDG methods are of order $k$ when polynomials of degree $k$ are used. However, their numerical experiments indicate that the order of convergence varies with the definition of the numerical fluxes and that a simple choice gives the optimal rate of $k+1$; in this volume, Castillo [41] gives a proof of this fact.

The elliptic case. It must be pointed out that when applied to elliptic problems, the LDG method can be ill-posed if the numerical fluxes are not carefully chosen; this happens, for example, for the fluxes chosen by Giannakouros and by Bassi and Rebay for their original DG scheme. This difficulty was overcome in 1997 by Bassi, Rebay, Mariotti, Pedinotti, and Savini [28] by means of a suitable modification of their original DG scheme; the resulting scheme was then further developed in 1998 by Bassi and Rebay [27]. At the same time, Brezzi, Manzini, Marini, Pietra and Russo [38] analyzed this problem and found several modifications resulting in well posed numerical methods for which they proved optimal error estimates; the scheme developed in [28] and [27] is one of these methods.

\subsection{Numerical experiments: Compressible Navier-Stokes}

From now on, as is customary in the finite element community, we use $p$ instead of $k$ to denote the degree of polynomials. The numerical results we show next are from Lomtev and Karniadakis [123].

Transonic flow past an airfoil. First we consider a refinement study for a transonic flow past an airfoil NACA0012 at an angle of attack $\alpha=10^{\circ}$, freestream Mach number $M a=0.8$, and Reynolds number based on the freestream velocity and the airfoil chord equal to $R e=73$. The wall temperature is equal to the freestream total temperature. The same problem is considered in [25] and is one of the benchmark problems suggested in the GAMM (1986) workshop [94]. The mesh is shown in Fig. 6; it extends 4 chords downstream and consists of 592 elements, which is about one-fourth of the number used in [25]. Three different discretizations with $p$-refinement were used corresponding to order 2,4 and 6 . The maximum order used in [25] 
was 3. In Fig. 7, we plot Mach contours for the first two discretizations ( $p=2$ and 4) that show the improvement in the solution as the polynomial order is increased. A more quantitative comparison is shown in Table 1 where we present the drag and lift coefficients for the three meshes; very good agreement with the results of [25] is obtained. The same is true for the distribution of the pressure and friction coefficients around the airfoil as shown in Fig. 8.

Table 1. Drag and lift coefficients corresponding to different $p$-refinements.

\begin{tabular}{cccc}
\hline Item & $p=2$ & $p=4$ & $p=6$ \\
\hline$C_{d}$ & 0.68287 & 0.67858 & 0.6758 \\
$C_{l}$ & 0.47625 & 0.53022 & 0.53173 \\
\hline
\end{tabular}

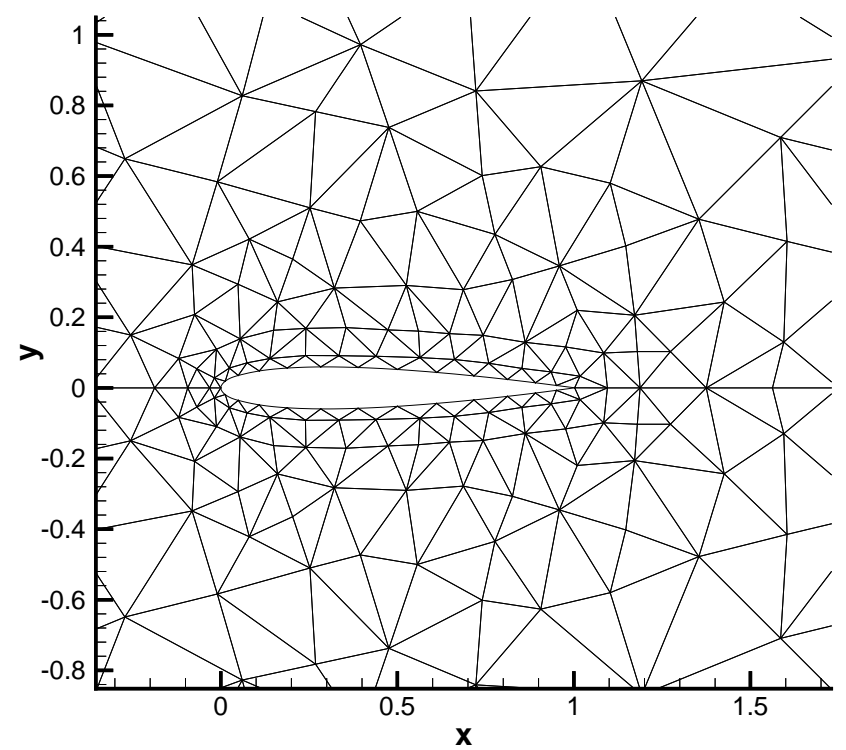

Fig. 6. Discretization around a NACA0012 airfoil; 592 elements are used.

Supersonic flow past an airfoil. We now consider a supersonic flow past a NACA 4420 airfoil at Mach number 2 and Reynolds number (based on 

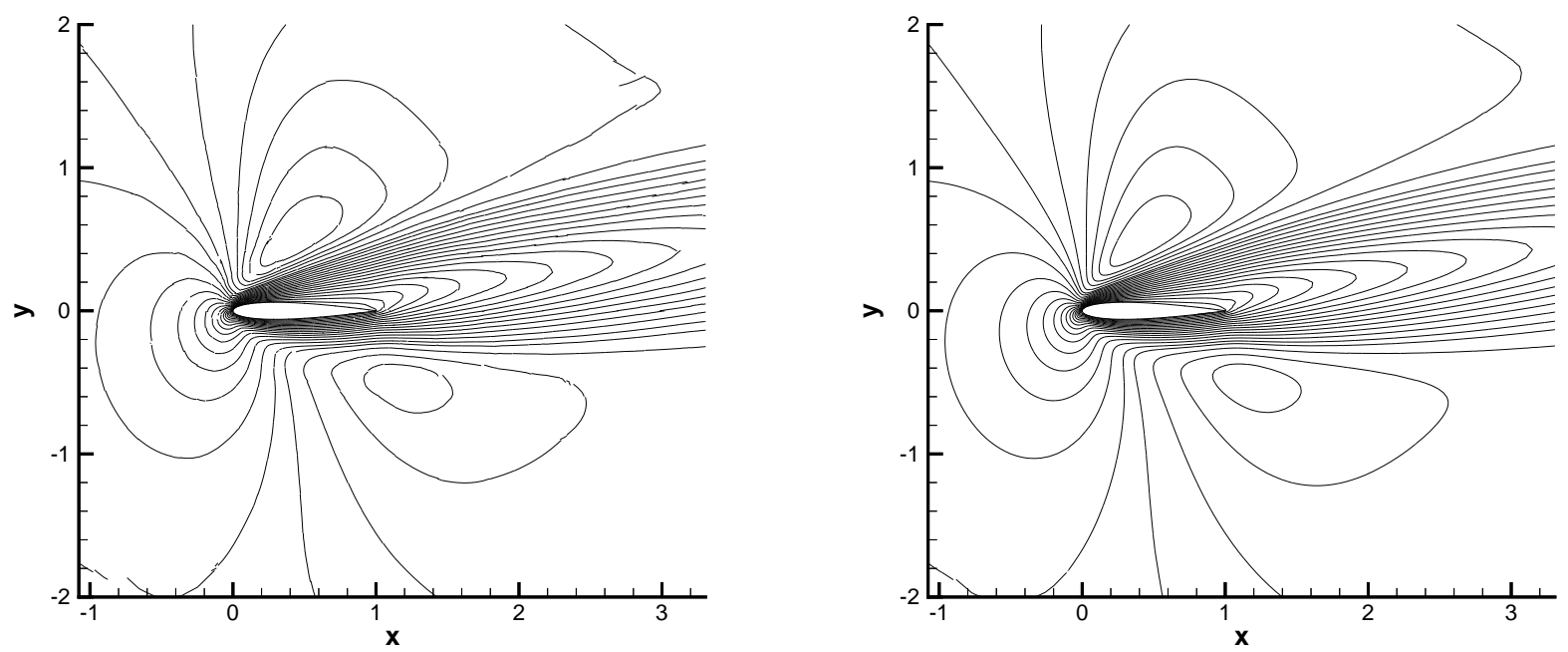

Fig. 7. Mach contour lines for discretization with $p=2$ (left) and $p=4$ (right).
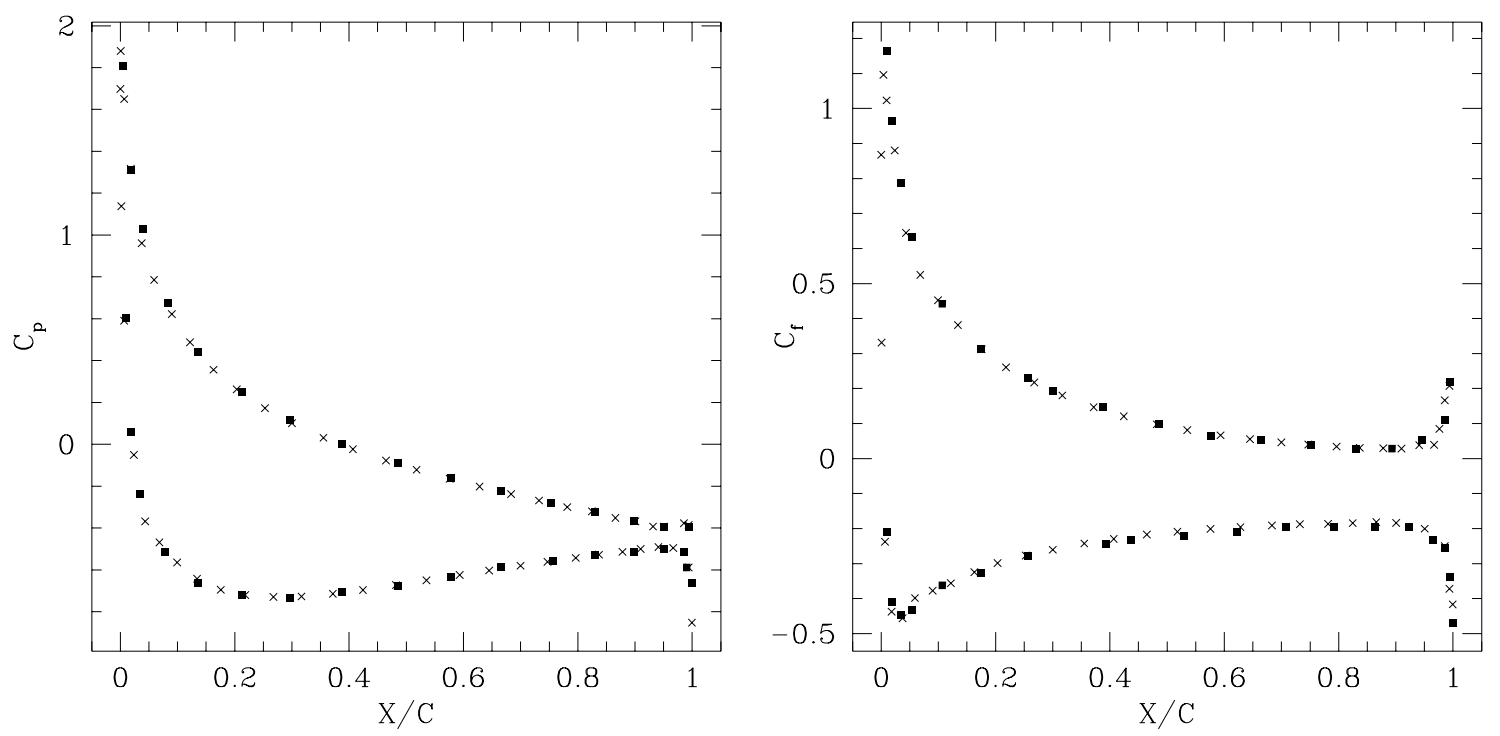

Fig. 8. Pressure (left) and drag (right) coefficients. Solid squares are data from [25] and crosses are from the current simulation for $p=6$. 
the chord length) 2400; the angle of attack is $20^{\circ}$. The domain extends from 1.25 chords upstream to 3.75 chords downstream and is discretized with 1492 triangles. Discretization and density contours and streamlines are shown in Fig. 9; the results are identical to earlier results obtained with results using a mixed formulation in [125]. Variable polynomial order is used from zero (constant elements) around the shock to $p=5$ in the wake. No flux limiters or filtering were used in this simulation.

\subsection{Numerical experiments: viscous MHD}

The numerical results we show next are from the work of Warburton and Karniadakis [171].

Simulation of the Orszag-Tang Vortex. We have performed a series of detailed simulations in order to investigate the small-scale structure exhibited in MHD turbulence. In particular, we consider a problem first studied by Orszag \& Tang (1979) [136] in the compressible case and later extended by Dahlburg \& Picone (1989) [62] to the compressible case. The initial conditions are non-random, periodic fields with the velocity field being solenoidal. The total initial pressure consists of the superposition of appropriate incompressible pressure distribution upon a flat pressure field corresponding to an initial average Mach number below unity. It was found in [136] and [62] that the coupling of the two-dimensional flow with the magnetic field causes the formation of singularities, i.e. excited small-scale structure, which although not as strong as the singularities in three-dimensional turbulence, they are certainly much stronger than two-dimensional hydrodynamic turbulence. Moreover, it was found in [62] that compressibility causes formation of additional small-scale structure such as massive jets and bifurcation of eddies. Our interest here is to investigate if we can capture these fine features both on structured and unstructured meshes, as shown in Fig 10.

The initial conditions we used were:

$$
\begin{aligned}
& \rho=1, \quad u=-\sin \left(\frac{2 \pi y}{L}\right), v=\sin \left(\frac{2 \pi x}{L}\right), B_{x}=-\sin \left(\frac{2 \pi y}{L}\right), B_{y}=\sin \left(\frac{4 \pi x}{L}\right), \\
& p=C+\frac{1}{4} \cos \left(\frac{8 \pi x}{L}\right)+\frac{4}{5} \cos \left(\frac{4 \pi x}{L}\right) \cos \left(\frac{2 \pi y}{L}\right)-\cos \left(\frac{2 \pi x}{L}\right) \cos \left(\frac{2 \pi y}{L}\right)+\frac{1}{4} \cos \left(\frac{4 \pi y}{L}\right),
\end{aligned}
$$

where $C$ fixes the initial average Mach number and $p$ is the instantaneous pressure for the equivalent incompressible flow.

We first simulate this MHD flow on a hybrid grid consisting of quadrilaterals and triangles as shown in Fig.10. We perform the simulations using the formulation of Powell [141] for the magnetic field as well as the streamfunction formulation with the objective of investigating divergence errors in the magnetic field. The rest of the parameters of this simulation are given in the 

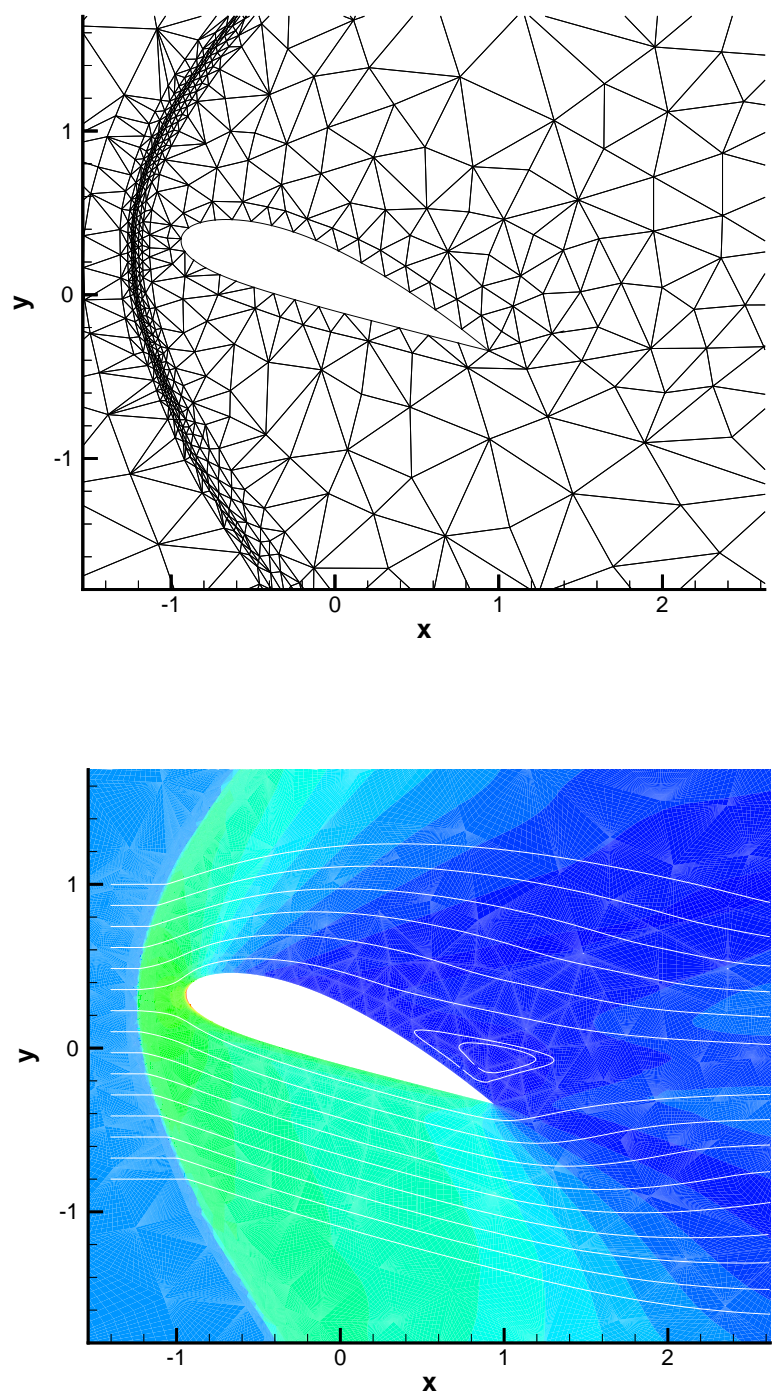

Fig. 9. Discretization around a NACA 4420 airfoil (top) and density contours and streamlines (bottom) at Mach number 2. 

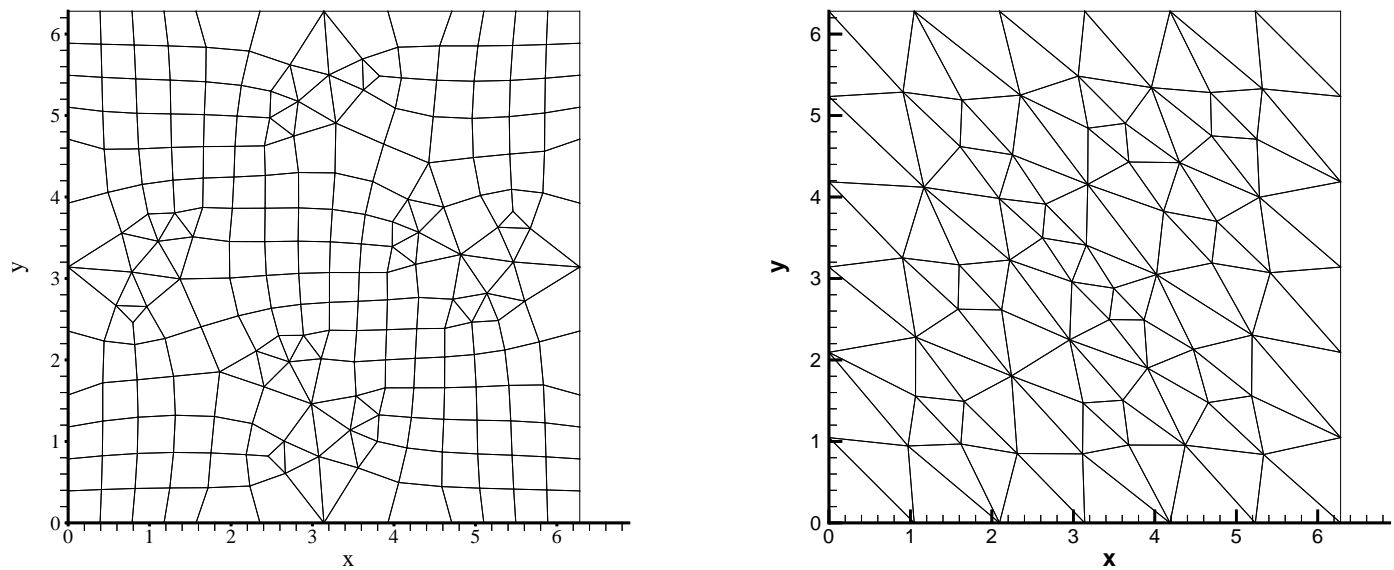

Fig. 10. Hybrid mesh on the left and unstructured mesh on the right used for the Orszag-Tang vortex simulations.

paper by Warburton and Karniadakis [171]. In Fig. 11, we plot streamlines of the incompressible flow as well as the compressible flow at Mach number 0.4 and non-dimensional time $t=2.0$. These results agree very well with the simulations of [62] at the same set of parameters. We note here that the compressible flow exhibits structures of finer features compared to the incompressible flow but the differences in the magnetic field are less obvious.

\subsection{Baumann-Oden DG method}

In 1998, Baumann and Oden [29] introduced a new DG method for the discretization of second-order problems; see also the paper by Oden, Babuška and Baumann [133]. Since the method is not a mixed method, it results in fewer degrees of freedom per element, a property that may make it competitive in Navier-Stokes approximations. For diffusion problems, this method is stable when polynomials of degree greater or equal to 2 ; adaptive $h p$-versions for Navier-Stokes equations have been implemented which exhibit exponential convergence rates. In this volume, Oden and Baumann [135] consider their method for convection-diffusion and the Navier-Stokes equations; see also the work done in 1998 by Baumann and Oden in [30]. In this volume, Arnold, Brezzi, Cockburn and Marini [8] propose a unified framework which contains almost all DG methods for elliptic equations including the Baumann-Oden method.

In this volume, Béatrice Rivière and Mary Wheeler [150] presents an error analysis of three interior penalty methods, some of which are related 

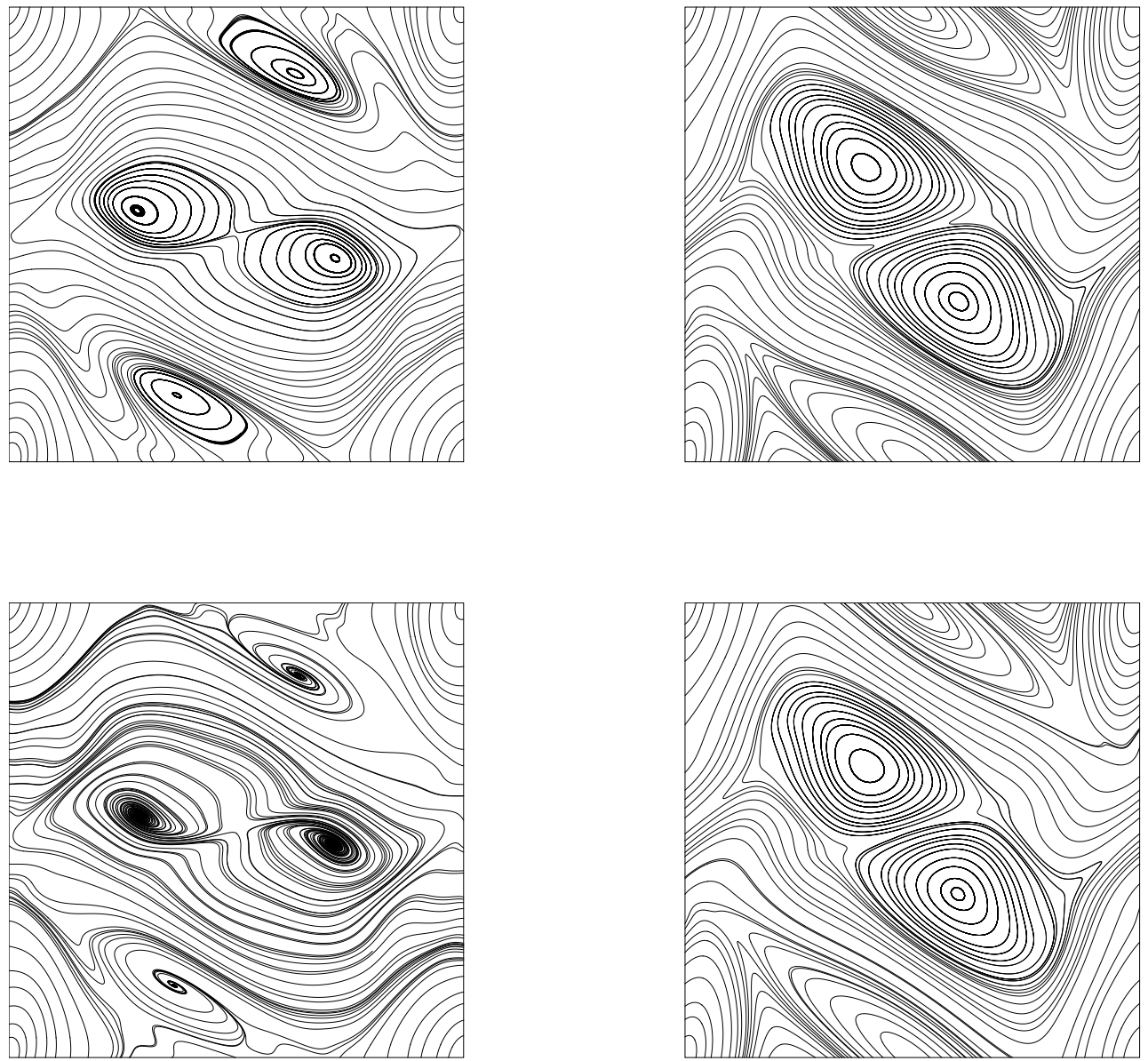

Fig. 11. Compressible Orszag-Tang vortex ( $\mathrm{t}=2$, instantaneous fields, Mach $=0.4$ ). Top: Incompressible flow; Left: Flow streamlines; Right: Magnetic Streamlines. Bottom: Compressible flow; Left: Flow streamlines; Right: Magnetic Streamlines. 
to the Baumann and Oden method. Also in this volume, an extension of the error analysis of the Baumann-Oden DG method to partial differential equations with non-negative characteristic form, is presented by Süli, Schwab, and Houston [162].

\subsection{New developments and applications}

In this volume, there are several new contributions to the development of DG methods for convection-diffusion problems:

- Bassi [24] reviews his recent work on the high-order, implicit, DG solution of the Reynolds Averaged Navier-Stokes equations coupled with the komega turbulence model closure; Rebay [144] shows how to use a GMRES solver in conjunction with the DG method for the compressible NavierStokes equations; and Liu and Shu [122] consider the use of the DG method for $2 \mathrm{D}$ incompressible flows.

- Prasad, Milovich, Shestakov, Kershaw, and Shaw [142] present a 3D unstructured ALE hydrodynamic DG method; and Lomtev, Kirby, and Karniadakis [124] introduce a discontinuous Galerkin ALE method for compressible flows in moving domains.

- Dawson, Aizinger and Cockburn [68] apply the LDG method to contaminant transport; Schwanenberg and Kongeter [153] use the method for shallow water equations; and Carranza, Fang, and Haber [40] introduce an adaptive DG method for coupled viscoplastic crack growth and chemical transport.

- Atkins [10] outlines the construction of a robust, high-order simulation tool for aerospace applications.

\section{Hamilton-Jacobi and second-order nonlinear equations}

\subsection{The method}

Recently, Hu and Shu [104] extended both the RKDG and the LDG method to the Hamilton-Jacobi equation

$$
u_{t}+H(D u)=f,
$$

and to the general nonlinear second-order differential equation

$$
u_{t}+F\left(u, D u, D^{2} u\right)=f
$$

for which the mapping $r \mapsto F(r, \cdot, \cdot)$ is increasing and $F(\cdot, \cdot, X) \geq F(\cdot, \cdot, Y)$ provided that $X \leq Y$.

The main idea is to exploit the equivalence of the Hamilton-Jacobi equation with the conservation law systems satisfied by the gradient of the solution and utilize the advantage of finite elements in maintaining the solution 
(not individual components of its gradient) as piecewise polynomials. A least square procedure is used to apply the discontinuous Galerkin framework to the conservation law system satisfied by the gradients, and the solution is recovered from its gradients by using again the Hamilton-Jacobi equation. Numerical results indicate that it is very important to keep the solution itself a polynomial and use the least square procedure.

\subsection{Numerical experiment: Motion by mean curvature}

To illustrate the performance of the DG method, we consider the initial value problem for the following nonlinear second-order differential equation:

$$
\begin{aligned}
& \varphi_{t}-(1-\varepsilon K) \sqrt{1+\varphi_{x}^{2}+\varphi_{y}^{2}}=0, \quad 0<x<1,0<y<1, \\
& \varphi(x, y, 0)=1-\frac{1}{4}(\cos (2 \pi x-1))(\cos (2 \pi y-1)),
\end{aligned}
$$

where $K$ is the mean curvature defined by

$$
K=-\frac{\varphi_{x x}\left(1+\varphi_{y}^{2}\right)-2 \varphi_{x y} \varphi_{x} \varphi_{y}+\varphi_{y y}\left(1+\varphi_{x}^{2}\right)}{\left(1+\varphi_{x}^{2}+\varphi_{y}^{2}\right)^{\frac{3}{2}}},
$$

and $\varepsilon$ is a small constant. Periodic boundary conditions are imposed. This problem was studied in 1998 by Osher and Sethian [137] by using the finite difference ENO schemes.

We can see that the resolution is excellent even without using any limiters. The singularity of the solution is captured sharply without noticeable oscillations.

\subsection{New developments}

In this volume, $\mathrm{Hu}$, Lepsky and Shu [103] present a study of the least square procedure for DG methods for Hamilton-Jacobi equations. Also, Augoula and Abgrall [12] develop a new algorithm for Hamilton-Jacobi equations.

\section{Parallelization, adaptivity and implementational issues}

\subsection{Parallelization and adaptivity}

Parallelization strategies for the steady-state transport equation were developed in 1995 by Bey, Patra, and Oden [35] and in 1996 by Bey, Oden and Patra [34]. They were based on the $1996 \mathrm{hp}$ - a posteriori error estimate obtained by Bey and Oden [33].

In 1994, Biswas, Devine, and Flaherty [36] carried out the first study of parallelization and adaptivity for RKDG methods for nonlinear conservation laws; a remarkable feature of this study is the use of their generalized slope 

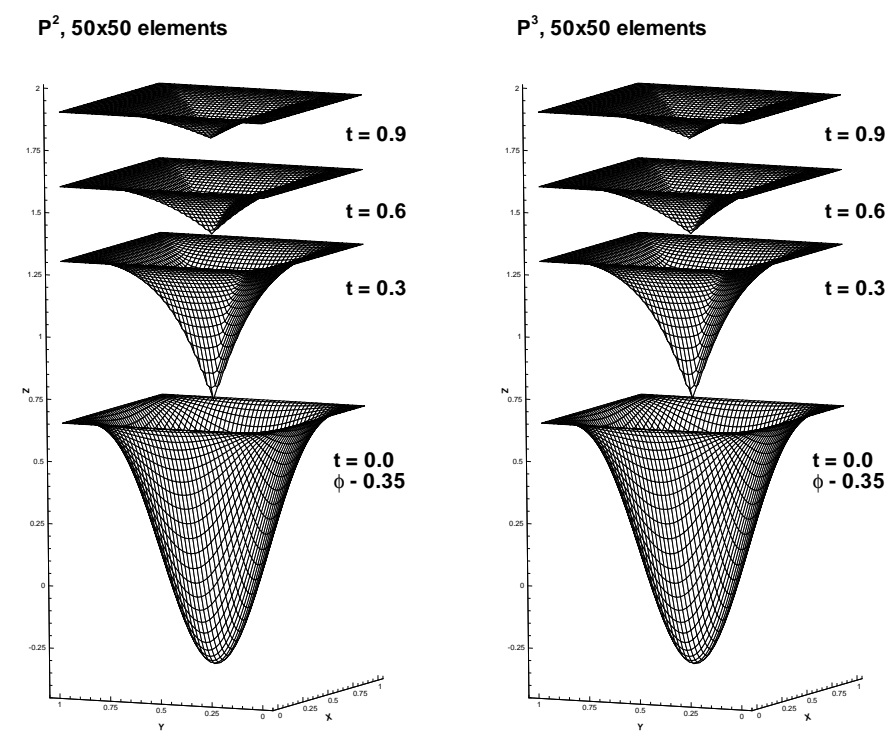

Fig. 12. Propagating surfaces, rectangular mesh, $\varepsilon=0$.

limiter to perform adaptive limiting. In 1996, Devine and Flaherty [73] devised a parallel adaptive $h p$-refinement techniques for hyperbolic systems in 2 space dimensions; a local time-stepping was used. The crucial issue of load balancing, which addresses the tension between parallelization and adaptivity, was considered in 1993 by Devine, Flaherty, Wheat, and Maccabe [75] for two-space dimension problems, and for three-space dimension problems in 1994 by deCougny, Devine, Flaherty, Loy, and Ozturan [69], and by Ozturan, deCougny, Shephard, and Flaherty [138], and in 1995 by Devine, Flaherty, Loy, and Wheat [74]. Parallel strategies, like predictive load-balancing, with local time-stepping techniques in the three-dimensional case have been devised and tested in 1997 by Flaherty, Loy, Shephard, Szymanski, Teresco, and Ziantz [91], in 1998 by Flaherty, Loy, Özturan, Shephard, Szymanski, Teresco and Ziantz [89], and in 1999 by Flaherty, Loy, Shephard, Simone, Szymanski, Teresco and Ziantz [90]. Recently, Teresco, Beall, Flaherty, and Shephard [165] extended this work and developed the TRELLIS framework and the RPM parallel data management. In this volume, Flaherty, Loy, Shephard and Teresco [88] report on the newest development of this technique.

In this volume, Aharoni and Barak [4] present an iterative, asynchronous parallel algorithm for PDEs using DG discretizations. 

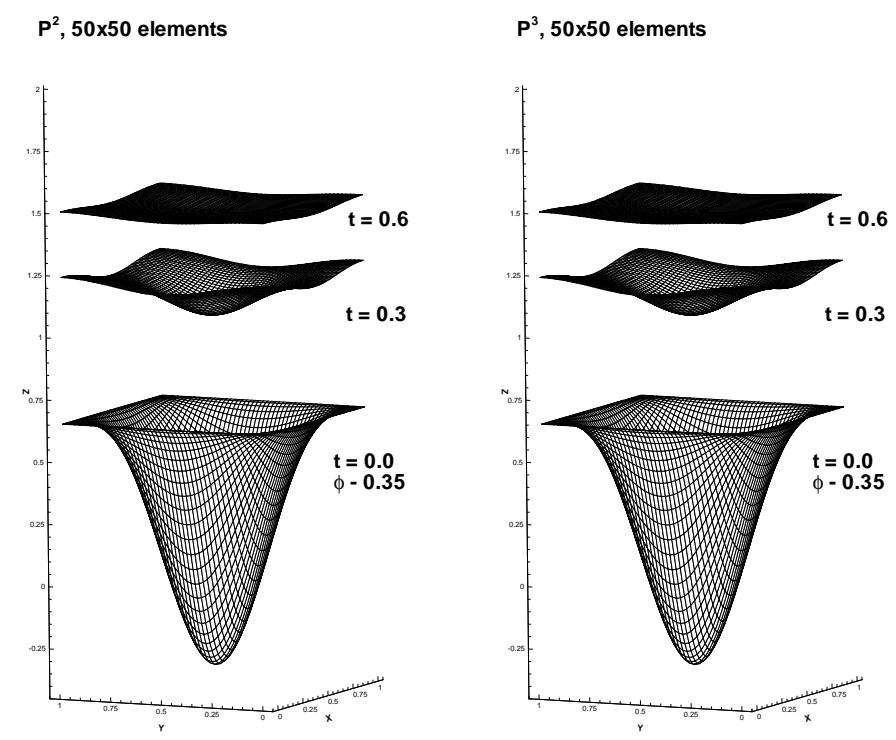

Fig. 13. Propagating surfaces, rectangular mesh, $\varepsilon=0.1$.

\subsection{Spectral/hp Element Methods - The Basis}

DG methods are particularly efficient when they are combined with highorder discretization. To this end, Karniadakis and Sherwin [114] and Warburton [170] have developed a hierarchical tensor-type basis extending the original ideas of Dubiner [76]. This basis is appropriate for hybrid discretizations, it is a combination of structured and unstructured domains consisting of polymorphic subdomains; for example, tetrahedra, hexahedra, triangular prisms, and pyramids. For each of these subdomains they have developed a polynomial expansion based upon a new local co-ordinate system [114]. These expansions are polynomials in terms of the local co-ordinates as well as the Cartesian co-ordinates $\left(\xi_{1}, \xi_{2}, \xi_{3}\right)$. This is a significant property as primary operations such as integration and differentiation can be performed with respect to the local co-ordinates but the expansion may still be considered as a polynomial expansion in terms of the Cartesian system.

An important property is that these expansions are orthogonal in the Legendre inner product. To wit, we define three principle functions $\phi_{i}^{a}(z), \phi_{i j}^{b}(z)$ and $\phi_{i j k}^{c}(z)$ in terms of the Jacobi polynomial $P_{p}^{\alpha, \beta}(z)$ as:

$$
\phi_{i}^{a}(z)=P_{i}^{0,0}(z), \quad \phi_{i j}^{b}(z)=\left(\frac{1-z}{2}\right)^{i} P_{j}^{2 i+1,0}(z)
$$




$$
\phi_{i j k}^{c}(z)=\left(\frac{1-z}{2}\right)^{i+j} P_{k}^{2 i+2 j+2,0}(z) .
$$

Using these functions we can construct the orthogonal polynomial expansions:

Hexahedral expansion: Prismatic expansion:

$$
\phi_{p q r}\left(\xi_{1}, \xi_{2}, \xi_{3}\right)=\phi_{p}^{a}\left(\xi_{1}\right) \phi_{q}^{a}\left(\xi_{2}\right) \phi_{r}^{a}\left(\xi_{3}\right) \quad \phi_{p q r}\left(\xi_{1}, \xi_{2}, \xi_{3}\right)=\phi_{p}^{a}\left(\xi_{1}\right) \phi_{q}^{a}\left(\eta_{2}\right) \phi_{q r}^{b}\left(\xi_{3}\right)
$$

Pyramidic expansion:

Tetrahedral expansion:

$\phi_{p q r}\left(\xi_{1}, \xi_{2}, \xi_{3}\right)=\phi_{p}^{a}\left(\overline{\eta_{1}}\right) \phi_{q}^{a}\left(\eta_{2}\right) \phi_{p q r}^{c}\left(\eta_{3}\right) \phi_{p q r}\left(\xi_{1}, \xi_{2}, \xi_{3}\right)=\phi_{p}^{a}\left(\eta_{1}\right) \phi_{p q}^{b}\left(\eta_{2}\right) \phi_{p q r}^{c}\left(\eta_{3}\right)$

where

$\eta_{1}=\frac{2\left(1+\xi_{1}\right)}{\left(-\xi_{2}-\xi_{3}\right)}-1, \quad \overline{\eta_{1}}=\frac{2\left(1+\xi_{1}\right)}{\left(1-\xi_{3}\right)}-1, \quad \eta_{2}=\frac{2\left(1+\xi_{2}\right)}{\left(1-\xi_{3}\right)}-1, \quad \eta_{3}=\xi_{3}$,
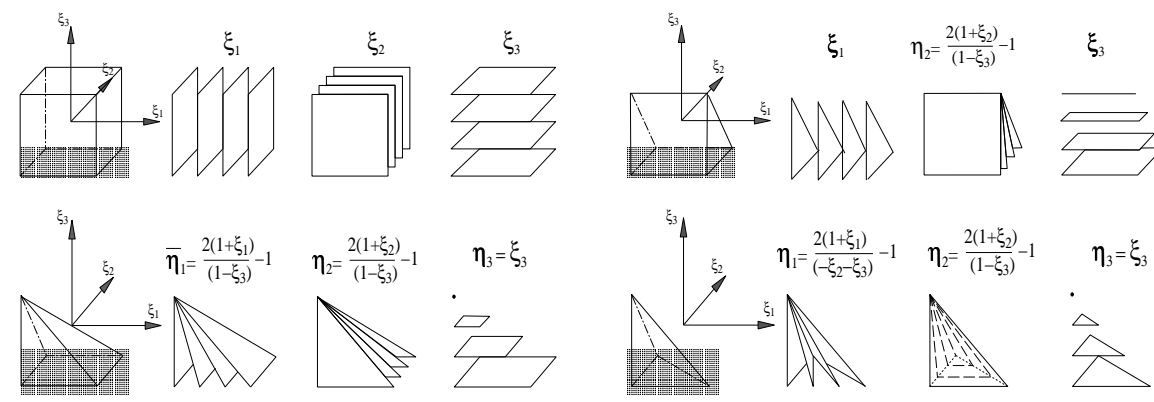

Fig. 14. The local coordinates $\left(\xi_{1}, \xi_{2}, \xi_{3}\right)$.

The hexahedral expansion is simply a standard tensor product of Legendre polynomials (since $P_{p}^{0,0}(z)=L_{p}(z)$ ). In the other expansions the introduction of the degenerate local co-ordinate systems is linked to the use of the more unusual functions $\phi_{i j}^{b}(z)$ and $\phi_{i j k}^{c}(z)$. Both these functions contain factors of the form $\left(\frac{1-z}{2}\right)^{p}$ which is necessary to keep the expansion a polynomial of the Cartesian co-ordinates $\left(\xi_{1}, \xi_{2}, \xi_{3}\right)$. For example, the co-ordinate $\eta_{2}$ in the prismatic expansion necessitates the use of the function $\phi_{q r}^{b}\left(\xi_{3}\right)$ which introduces a factor of $\left(\frac{1-\xi_{3}}{2}\right)^{q}$. The product of this factor with $\phi_{q}^{a}\left(\eta_{2}\right)$ is a polynomial function in $\xi_{2}$ and $\xi_{3}$. Since the remaining part of the prismatic expansion, $\phi_{p}^{a}\left(\xi_{1}\right)$, is already in terms of a Cartesian co-ordinate, the whole expansion is a polynomial in terms of the Cartesian system.

The polynomial space, in Cartesian co-ordinates, for each expansion is:

$$
\mathcal{P}=\operatorname{Span}\left\{\xi_{1}^{p} \xi_{2}^{q} \xi_{3}^{r}\right\}
$$


where $p q r$ for each domain is

$$
\begin{array}{lll}
\text { Hexahedron } & 0 \leq p \leq P_{1}, 0 \leq q \leq P_{2}, & 0 \leq r \leq P_{3}, \\
\text { Prism } & 0 \leq p \leq P_{1}, 0 \leq q \leq P_{2}, & 0 \leq q+r \leq P_{3}, \\
\text { Pyramidic } 0 \leq p \leq P_{1}, 0 \leq q \leq P_{2}, & 0 \leq p+q+r \leq P_{3}, \\
\text { Tetrahedron } 0 \leq p \leq P_{1}, 0 \leq p+q \leq P_{2}, 0 \leq p+q+r \leq P_{3}
\end{array}
$$

The range of the $p, q$ and $r$ indices indicate how the expansions should be formed to generate a complete polynomial space. We note that if $P_{1}=P_{2}=$ $P_{3}$ then the tetrahedral and pyramidic expansions span the same space and are in a subspace of the prismatic expansion which is in turn a subspace of the hexahedral expansion.

An important property of the hybrid spectral basis is that it is orthogonal in the new coordinate system. This simplifies greatly the discontinuous Galerkin formulation, since all mass matrices are diagonal and their inversion is trivial.

\subsection{Quadrature-free implementations}

In 1998, Atkins and Shu [11] introduced the first quadrature-free implementation of the RKDG and LDG methods. The idea is to use an easily manipulated local basis, such as the local basis used in the Taylor expansions at the center of the cell, and expand the nonlinear terms in a (suitably truncated) polynomial in this local basis based on the solution itself. The integration of products of polynomials in this local basis can be precomputed and stored, in fact a similarity transformation allows one to only store extensive data for one reference object in each class of elements (triangles, quadralaterals, elements with curved boundaries, etc.). Significant speed up can be obtained for linear problems and simple nonlinear problems such as Euler equations with

only multiplicative nonlinearity and one division (by density). The coding structure is also simplified in this formulation.

\subsection{Object-oriented implementations}

Several object-oriented implementations of DG methods have already been developed. The code NEKTAR (freeware), developed at Brown University, is written in $\mathrm{C}++$ and MPI for parallel implementation, is being currently used in more than twenty universities, national laboratories, and industries. As pre-processor, it uses the code FELISA [139] to generate 2D and 3D grids and METIS [115] for parallel domain decomposition. Specifically, to obtain the partition of the $3 \mathrm{D}$ mesh for simulation of compressible Navier-Stokes and viscous MHD, NEKTAR uses a multi-level graph theoretical approach, similar to the one used in METIS, which, however, takes into account the $p$-modes on each element by using appropriate weights. The code allows for a variable polynomial order per element and for all different shapes of elements 
including tetrahedra, hexahedra, triangular prisms, and pyramids using the tensor-product Jacobi basis described earlier. This discretization flexibility is useful for complex geometry simulations, especially for viscous compressible and MHD flows and leads to large parallel efficiencies due to the high volumeover-surface ratio associated with the $p$-expansions.

The TRELLIS framework and the RPM data management system are also object-oriented implementations; see the 1998 paper by Teresco, Beall, Flaherty and Shephard [165].

In this volume, Atkins [10] reports on efforts towards the contruction of a high-order simulation tool for aerospace applications based on DG methods. Also, Prasad, Milovich, Shestakov, Kershaw and Shaw [142] describe a 3D ALE version of a DG method for solving hydrodynamic problems relevant to inertial confinement fusion.

\section{Open problems and concluding remarks}

\subsection{Open problems and future developments}

One of the main challenges for the development of finite element methods is the construction and analysis of efficient techniques for problems in computational fluid dynamics. In what follows, we discuss open questions and future developments for one of those methods, the DG methods.

Local conservativity. It is well known that practioners in the area of numerics for nonlinear conservation laws, overwhelmingly prefer locally conservative numerical schemes. This is not the case for elliptic or parabolic equations, however, for which the widely used classical finite element methods are not locally conservative. These points of view clearly clash when the issue of how to approximate convection-diffusion problems arises. A deep analysis of these two properties constitutes a very interesting open problem. An effort in this direction can be found in this volume in the paper by Hughes, Engel, Mazzei, and Larson [105].

Slope limiters. An important component of the RKDG method for transient nonlinear hyperbolic systems is the generalized slope limiter. Although this slope limiter does not seem to be needed in computations involving diffusive flows, it is necessary for the current DG methods for purely hyperbolic problems. The slope limiter used in the RKDG methods involve a parameter (which in one-dimensional scalar conservation laws is nothing but an upper bound of the second-order derivative of the solution at critical points) by means of which the limiting does not destroy accuracy at critical points. An efficient way of estimating this parameter in terms of the computed approximate solution remains to be obtained. Another challenging problem is how to devise a slope limiter that is free from such a parameter. Finally, since the 
slope limiter is computationally expensive, it would be very useful to devise a DG method that does not have a slope limiter and remains nonlinearly stable and high-order accurate.

Time-steppping. High-order accurate time-discretizations which are capable of treating the convective terms explicitly and the diffusive term implicitly, if necessary, have not been developed yet and are in high demand nowadays.

Also, in order to be able to do adaptivity while maintaining the high parallelizability of the DG methods, new high-order accurate time-stepping methods would have to be created which could use different time steps at different locations. The use of space-time DG methods could be a possible way of overcoming this difficulty, but they tend to be rather difficult to code- and are not very efficient. Another possibility is to extend to high-order accurate schemes the approach used in 1995 by Dawson [65] to devise a first-order accurate, conservative variable time-stepping schemes. Non-conservative timestepping methods can also lead to efficient time-discretizations, but one has to be very careful to exert a tight control on the loss of mass, especially near the discontinuities. A very interesting example is the local time stepping technique introduced in 1997 by Flaherty, Loy, Shephard, Szymanski, Teresco, and Zianz [91].

Quadrature crimes and over-integration. In 1998, Atkins and Shu [11] introduced a quadrature-free implementation of the RKDG method. They used truncated expansions of the nonlinear integrands that could then be evaluated exactly. A challenging problem is to determine the way the above mentioned expansions have to be truncated to ensure both stability and accuracy of the resulting DG method.

In this volume, Lomtev, Kirby, and Karniadakis [124] show that, in order to produce high-quality approximations, over-integration of one or even two extra degrees of accuracy is necessary when steep gradients on the approximate solution appear near the boundary. Although the LDG method have been proven to be stable, even for nonlinear convection, see [59], such result assumes exact integration. A systematic study of the dependence of the stability of the LDG method for nonlinear convection with respect to the quadrature rules is an interesting open problem. For elliptic equations, work in this direction was pursued in 1990 by Maday and Ronquist [131] on the $h p$-Galerkin/spectral method.

Approximation of singularities. It is very well known that singularities often appear in nonlinear and even linear flows. In the past, to deal with those, $a d$ hoc strategies have been employed. An example is the idea introduced in 1984 by Woodward and Colela [178] to deal with the corner singularity of the forward facing step test problem. In 1998, Cockburn and Shu [60] showed that to deal with that singularity, it is enough to simply refine the mesh 
around the corner, as it is customary in standard finite element methods. A significant effort towards a systematic handling of singularities, in particular in fluid dynamics, is being carried out by Schwab [151], [152].

Steady-state computations Efficient solvers for steady-state computations need to be developed; these solvers are also needed when implicit discretizations in time are used. In this volume, Rebay [144] shows how to use the GMRES for DG methods for compressible Navier-Stokes equations.

Error estimation. Several a priori and a posteriori error estimates are currently available for DG methods. For the linear case, see, for example, the review of a priori error estimates in the lecture notes of Cockburn [47], [48]; for a posteriori error estimates, see the lecture notes of Süli [159], [160]. See also the recent papers by Falk and Richter [87], by Houston, Schwab, and Süli [102] and the paper in this volume by Süli, Schwab, and Houston [162]. For the nonlinear case, see the 1996 paper by Cockburn and Gremaud [50] and the 1999 lecture notes by Cockburn [49]. This is a rapidly developing area that deserves special attention since its development will lead to computations with a preassigned, guaranteed accuracy. Refinements of the above mentioned results and adaptivity strategies based on them which fully take advantage of the use of discontinuous approximations will be developed in the coming years.

Super-convergence. In 1994, Biswas, Devine, and Flaherty [36] gathered numerical evidence that, when rectangular elements are used, the approximate solution of the DG method super-converges at the Gauss-Radau points and exploited this for adaptivity purposes. This fact was recently proven [3]; see also the papers by Adjerid, Aiffa and Flaherty [2] and [1]. The search for super-convergence points in simplexes remains an interesting open problem. Also, the way to exploit the super-convergence of the postprocessed solution obtained by Cockburn, Luskin, Shu and Süli [54] for adaptivity purposes remains a challenging open problem.

Multiresolution analysis. The incorporation of multiresolution analysis into the finite element method is an exciting undertaking (which has to be differentiated from the standard finite element $h p$-refinement). One of the main difficulties is devising of wavelets satisfying boundary conditions, but with the use of the DG method this is no longer a requirement. As a consequence, the use of wavelet-based discontinuous Galerkin methods constitutes a possible breakthrough in this direction. In this volume, Coult [61] provides a most needed introduction to the subject. 


\section{Relation of the LDG method with other methods.}

- Mixed methods for elliptic equations. When applied to elliptic equations, the LDG method can be considered to be a mixed method. However, it was originally devised by using discretization techniques closer to convective problems rather that to elliptic ones. This is reflected, for example, in the fact that error estimates for LDG methods can be obtained without having to deal (explicitly) with the classical inf - sup condition! The relationship of the LDG method with standard and stabilized mixed methods (and their hybridization) for elliptic equations is still unexplored.

- Penalty methods for elliptic equations. The relation of the LDG method with the DG method of Baumann and with the interior penalty methods of Baker [15] (1977), Wheeler [176] (1978), Arnold [9] (1982), and Baker, Jureidini, and Karakashian [16] (1990), is another interesting open problem. In this volume, Arnold, Brezzi, Cockburn and Marini [8] propose a unified framework that includes almost all the numerical methods proposed for elliptic equations that use totally discontinuous finite element discretizations.

- Upwind-Mixed Methods The relation between the Upwind-Mixed Methods introduced by Dawson [63], [64], [65], [66], and [67] and the LDG methods remains unexplored. In this volume, a first step towards a thorough comparison of these methods is presented by Dawson, Aizinger, and Cockburn [68]. An interesting point is to find out if the use of discontinuous discretizations of second-order terms have any advantage over the classical mixed finite element approximations.

- Streamline diffusion methods. The relationship between DG methods and streamline-diffusion methods is quite close but has never been studied. For example, Cockburn and Gremaud [50] analyzed these two methods as applied to the nonlinear scalar conservation law with the same technique. See the work of Houston, Schwab and Süli [102] in this direction. Also, there is a close relationship between the generalized slope limiters that some DG methods use and the so-called shock-capturing terms embedded in the definition of the streamline diffusion methods; this relation is still unexplored. An effort in this direction is the paper in this volume by Hughes, Engel, Mazzei, and Larson [105] in which a comparison of discontinuous and continuous methods is offered.

- The cell discretization method. In this volume, Greenstadt [97] and Swann [164] review their work on the so-called cell discretization method. This is a very interesting method related to nonconforming methods for elliptic equations and possibly to some DG methods.

\subsection{Conclusion}

Let us conclude this review by saying that the future development of DG methods which will take place in the next few years is an exciting scientific undertaking. Witnesses to the rapid incorporation of the finite element 
methodology in computational fluid dynamics are the books by Schwab [151] and Sherwin and Karniadakis [114]; see also the lecture notes of Cockburn [47], [48], Schwab [152], and Süli [159], [160].

Acknowledments. We would like to acknowledge the support of NSF, DOE and ARO for the organization of this First International Symposium on Discontinuous Galerkin Methods. We would also like to thank Francesco Bassi, Igor Lomtev, Stefano Rebay, and Tim Warburton for permitting us to use their plots; and to S.R. Allmaras, C. Baumann, J. Flaherty, R. Haber, C. Johnson, K.W. Morton, T. Oden, and E. Süli for their valuable feedback on the first version of this review. The research work of the first author is supported by NSF and the Minnesota Supercomputing Institute, that of the second author by AFOSR and DOE, and that of the third author by ARO, NSF, NASA, and AFOSR.

\section{References}

1. S. Adjerid, M. Aiffa, and J. E. Flaherty, Computational methods for singularly perturbed systems, Singular Perturbation Concepts of Differential Equations (J. Cronin and R.E. O'Malley, eds.), AMS Proceedings of Symposia in Applied Mathematics, AMS, 1998.

2. S. Adjerid, M. Aiffa, and J.E. Flaherty, High-order finite element methods for singularly-perturbed elliptic and parabolic problems, SIAM J. Appl. Math. 55 (1995), 520-543.

3. S. Adjerid, J.E. Flaherty, and L. Krivodonova, Superconvergence and a posteriori error estimation for continuous and discontinuous Galerkin methods applied to singularly perturbed parabolic and hyperbolic problems, in preparation.

4. D. Aharoni and A. Barak, Parallel iterative discontinuous Galerkin FEM, this volume, 1999, pp. - .

5. S.R. Allmaras, A coupled Euler/Navier Stokes algorithm for 2-D unsteady transonic shock/boundary-layer interaction, Ph.D. thesis, Massachussetts Institute of Technology, 1989.

6. S.R. Allmaras and M.B.Giles, A second order flux split scheme for the unsteady 2-D Euler equations on arbitrary meshes, 8th. AIAA Computational Fluid Dynamic Conference, Honolulu, Hawai, June 9-11, 1987, AIAA 87-1119-CP.

7. _ A coupled Euler/Navier-Stokes algorithm for 2-D transonic flows, 27th. Aerospace Sciences Meeting, Reno, Nevada, January 9-12, 1989.

8. D. Arnold, F. Brezzi, B. Cockburn, and D. Marini, DG methods for elliptic problems, this volume, 1999, pp. -.

9. D.N. Arnold, An interior penalty finite element method with discontinuous elements, SIAM J. Numer. Anal. 19 (1982), 742-760.

10. H. Atkins, Steps toward a robust high-order simulation tool for aerospace applications, this volume, 1999, pp. -.

11. H.L. Atkins and C.-W. Shu, Quadrature-free implementation of discontinuous Galerkin methods for hyperbolic equations, AIAA Journal 36 (1998), 775-782.

12. S. Augoula and R. Abgrall, A discontinuous prjection algorith for HamiltonJacobi equations, this volume, 1999, pp. -. 
13. F.P.T. Baaijiens, A.C.B. Bogaerds, and W.M.H. Verbeeten, Successes and failures of discontinuous Galerkin methods in viscoelastic fluid analysis, this volume, 1999, pp. -.

14. A. Bahhar, J. Baranger, and D. Sandri, Galerkin discontinuous approximation of the transport equation and viscoelastic fluid flow on quadrilaterals, Numer. Methods Partial Differential Equations 14 (1998), 97-114.

15. G.A. Baker, Finite element methods for elliptic equations using nonconforming elements, Math. Comp. 31 (1977), 45-59.

16. G.A. Baker, W.N. Jureidini, and O.A. Karakashian, Piecewise solenoidal vector fields and the Stokes problem, SIAM J. Numer. Anal. 27 (1990), 1466-1485.

17. P. Bar-Yoseph, Space-time discontinuous finite element approximations for multidimensional nonlinear hyperbolic systems, Comput. Mech. 5 (1989), 145160.

18. P. Bar-Yoseph and D. Elata, An efficient $L^{2}$ Galerkin finite element method for multi-dimensional nonlinear hyperbolic systems, Internat. J. Numer. Methods Engrg. 29 (1990), 1229-1245.

19. J. Baranger and A. Machmoum, A "natural" norm for the discontinuous finite element characteristic method: the 1-D case, RAIRO Modél. Math. Anal.Numér. 30 (1996), 549-574.

20. Existence of approximate solutions and error bounds for viscoelastic fluid flow: Characteristics method, Comput. Methods Appl. Mech. Engrg. 148 (1997), 39-52.

21. J. Baranger and D. Sandri, Finite element approximation of viscoelastic fluid flow: existence of approximate solutions and error bounds. I. Discontinuous constraints., Numer. Math. 63 (1992), 13-27.

22. J. Baranger and S. Wardi, Numerical analysis of a FEM for a transient viscoelastic flow, Comput. Methods Appl. Mech. Engrg. 125 (1995), 171-185.

23. T. Barth, Simplified DG methods for systems of conservation laws with convex extension, this volume, 1999, pp. - .

24. F. Bassi, A high-order discontinuous Galerkin method for compressible turbulent flow, this volume, 1999, pp. -.

25. F. Bassi and S. Rebay, A high-order accurate discontinuous finite element method for the numerical solution of the compressible Navier-Stokes equations, J. Comput. Phys. 131 (1997), 267-279.

26. - High-order accurate discontinuous finite element solution of the $2 D$ Euler equations, J. Comput. Phys. 138 (1997), 251-285.

27. F. Bassi and S. Rebay, An implicit high-order discontinuous Galerkin method for the steady state compressible Navier-Stokes equations, Computational Fluid Dynamics 98, Proceedings of the Fourth European Computational Fluid Dynamics Conference (Athens, Greece) (K.D. Papailiou, D. Tsahalis, D. Périaux, C. Hirsh, and M. Pandolfi, eds.), vol. 2, John Wiley and Sons, September 5-7 1998, pp. 1227-1233.

28. F. Bassi, S. Rebay, G. Mariotti, S. Pedinotti, and M. Savini, A high-order accurate discontinuous finite element method for inviscid and viscous turbomachinery flows, 2nd European Conference on Turbomachinery Fluid Dynamics and Thermodynamics (Antwerpen, Belgium) (R. Decuypere and G. Dibelius, eds.), Technologisch Instituut, March 5-7 1997, pp. 99-108.

29. C.E. Baumann and J.T. Oden, A discontinuous $h p$ finite element method for convection-diffusion problems, Comput. Methods Appl. Mech. Engrg., in press, 
special issue on Spectral, Spectral Element, and $h p$ Methods in CFD, edited by G.E. Karniadakis, M. Ainsworth and C. Bernardi.

30. A discontinuous hp finite element method for the Navier-Stokes equations, 10th. International Conference on Finite Element in Fluids, 1998.

31. _ A discontinuous hp finite element method for the solution of the Euler equation of gas dynamics, 10th. International Conference on Finite Element in Fluids, 1998.

32. K.S. Bey and J.T. Oden, A Runge-Kutta discontinuous Galerkin finite element method for high speed flows, 10th. AIAA Computational Fluid Dynamics Conference, Honolulu, Hawaii, June 24-27, 1991.

33. tion laws, Comput. Methods Appl. Mech. Engrg. 133 (1996), 259-286.

34. K.S. Bey, J.T. Oden, and A. Patra, A parallel hp-adaptive discontinuous Galerkin method for hyperbolic conservation laws, Appl. Numer. Math. 20 (1996), 321-286.

35. K.S. Bey, A. Patra, and J.T. Oden, hp-version discontinuous Galerkin methods for hyperbolic conservation laws: A parallel strategy, Internat. J. Numer. Methods Engrg. 38 (1995), 3889-3908.

36. R. Biswas, K.D. Devine, and J. Flaherty, Parallel, adaptive finite element methods for conservation laws, Appl. Numer. Math. 14 (1994), 255-283.

37. K. Bottcher and R. Rannacher, Adaptive error control in solving ordinary differential equations by the discontinuous Galerkin method, Tech. report, University of Heidelberg, 1996.

38. F. Brezzi, D. Marini, P. Pietra, and A. Russo, Discontinuous finite elements for diffusion problems, Numerical Methods for Partial Differential Equations (1999), submitted.

39. W. Cai, Mixed high-order basis functions for electromagnetic scattering of curved surfaces, this volume, 1999, pp.--.

40. F.L. Carranza, B. Fang, and R.B. Haber, An adaptive discontinuous Galerkin model for coupled viscoplastic crack growth and chemical transport, this volume, 1999, pp. -.

41. P. Castillo, An optimal error estimate for the local discontinuous Galerkin method, this volume, 1999, pp. -

42. G. Chavent and B. Cockburn, The local projection $P^{0} P^{1}$-discontinuousGalerkin finite element method for scalar conservation laws, RAIRO Modél. Math. Anal.Numér. 23 (1989), 565-592.

43. G. Chavent and J. Jaffré, Mathematical models and finite elements for reservoir simulation, Studies in Mathematics and its Applications, vol. 17, NorthHolland, Amsterdam, 1986.

44. G. Chavent and G. Salzano, A finite element method for the $1 D$ water flooding problem with gravity, J. Comput. Phys. 45 (1982), 307-344.

45. Z. Chen, B. Cockburn, C. Gardner, and J. Jerome, Quantum hydrodynamic simulation of hysteresis in the resonant tunneling diode, J. Comput. Phys. 117 (1995), 274-280.

46. Z. Chen, B. Cockburn, J. Jerome, and C.-W. Shu, Mixed-RKDG finite element methods for the 2-D hydrodynamic model for semiconductor device simulation, VLSI Design 3 (1995), 145-158.

47. B. Cockburn, An introduction to the discontinuous Galerkin method for convection-dominated problems, Advanced numerical approximation of non- 
linear hyperbolic equations (A. Quarteroni, ed.), Lecture Notes in Mathematics; subseries Fondazione C.I.M.E., Firenze, vol. 1697, Springer Verlag, 1998, pp. $151-268$.

48. Discontinuous Galerkin methods for convection-dominated problems, High-Order Methods for Computational Physics (T. Barth and H. Deconink, eds.), Lecture Notes in Computational Science and Engineering, vol. 9, Springer Verlag, 1999, pp. 69-224.

49. — A simple introduction to error estimation for nonnlinear hyperbolic conservation laws. Some ideas, techniques, and promising results, Proceedings of the 1998 EPSRC Summer School in Numerical Analysis,SSCM, The Graduate Student's Guide to Numerical Analysis, vol. 26, Springer-Verlag, 1999, pp. $1-46$.

50. B. Cockburn and P.A. Gremaud, Error estimates for finite element methods for nonlinear conservation laws, SIAM J. Numer. Anal. 33 (1996), 522-554.

51. B. Cockburn, S. Hou, and C.W. Shu, TVB Runge-Kutta local projection discontinuous Galerkin finite element method for conservation laws IV: The multidimensional case, Math. Comp. 54 (1990), 545-581.

52. B. Cockburn, J. Jerome, and C.-W. Shu, The utility of modeling and simulation in determining performance and symmetry properties of semiconductors, this volume, 1999, pp. - .

53. B. Cockburn, S.Y. Lin, and C.W. Shu, TVB Runge-Kutta local projection discontinuous Galerkin finite element method for conservation laws III: One dimensional systems, J. Comput. Phys. 84 (1989), 90-113.

54. B. Cockburn, M. Luskin, C.-W. Shu, and E. Süli, Enhanced accuracy by postprocessing for finite element methods for hyperbolic equations, in preparation.

55. _ _ Postprocessing of Galerkin methods for hyperbolic problems, this volume, 1999, pp. - .

56. B. Cockburn and C.W. Shu, TVB Runge-Kutta local projection discontinuous Galerkin finite element method for scalar conservation laws II: General framework, Math. Comp. 52 (1989), 411-435.

57. - The $P^{1}-R K D G$ method for two-dimensional Euler equations of gas dynamics, Tech. Report 91-32, ICASE, 1991.

58. ㄴ The Runge-Kutta local projection $P^{1}$-discontinuous Galerkin method for scalar conservation laws, RAIRO Modél. Math. Anal.Numér. 25 (1991), $337-361$.

59. - The local discontinuous Galerkin finite element method for convection-diffusion systems, SIAM J. Numer. Anal. 35 (1998), 2440-2463.

60. _ The Runge-Kutta discontinuous Galerkin finite element method for conservation laws V: Multidimensional systems, J. Comput. Phys. 141 (1998), 199-224.

61. N. Coult, Wavelet-based discontinuous Galerkin methods, this volume, 1999, pp. -.

62. R.B. Dahlburg and J.M. Picone, Evolution of the Orszag-Tang vortex system in a compressible medium. I. Initial average subsonic flow, Phys. Fluids B 1 (1989), no. 11, 2153-2171.

63. C.N. Dawson, Godunov-mixed methods for advection-diffusion equations in one space dimension, SIAM J. Numer. Anal. 28 (1991), 1282-1309.

64. _ Godunov-mixed methods for advection-diffusion equations in multidimensions, SIAM J. Numer. Anal. 30 (1993), 1315-1332. 
65. _ High resolution upwind-mixed finite element methods for advectiondiffusion equations with variable time-stepping, Numerical Methods for Partial Differential Equations 11 (1995), 525-538.

66. _ Analysis of an upwind-mixed finite element method for nonlinear contiminant transport problems, SIAM J. Numer. Anal. 35 (1998), 1709-1724.

67. C.N. Dawson and V. Aizinger, Upwing-mixed methods for transport equations, Comp. Geo., to appear.

68. C.N. Dawson, V. Aizinger, and B. Cockburn, The Local Discontinuous Galerkin method for contaminant transport problems, this volume, 1999, pp.--.

69. H.L. deCougny, K.D. Devine, J.E. Flaherty, R.M. Loy, C. Ozturan, and M.S. Shephard, Load balancing for the parallel adaptive solution of partial differential equations, Appl. Numer. Math. 16 (1994), 157-182.

70. M. Delfour, W. Hager, and F. Trochu, Discontinuous Galerkin methods for ordinary differential equations, Math. Comp. 36 (1981), 455-473.

71. M. Delfour and F. Trochu, Discontinuous Galerkin methods for the approximation of optimal control problems governed by hereditary differential systems, Distributed Parameter Systems: Modelling and Identification (A. Ruberti, ed.), Springer Verlag, 1978, pp. 256-271.

72. B. Depres, Discontinuous Galerkin method for the numerical solution of euler equations in axisymmetric geometry, this volume, 1999, pp. -.

73. K.D. Devine and J.E. Flaherty, Parallel adaptive hp-refinement techniques for conservation laws, Appl. Numer. Math. 20 (1996), 367-386.

74. K.D. Devine, J.E. Flaherty, R.M. Loy, and S.R. Wheat, Parallel partitioning strategies for the adaptive solution of conservation laws, Modeling, mesh generation, and adaptive numerical methods for partial differential equations (I. Babuška, W.D. Henshaw, J.E. Hopcroft, J.E. Oliger, and T. Tezduyar, eds.), vol. 75, 1995, pp. 215-242.

75. K.D. Devine, J.E. Flaherty, S.R. Wheat, and A.B. Maccabe, A massively parallel adaptive finite element method with dynamic load balancing, Proceedings Supercomputing'93, 1993, pp. 2-11.

76. M. Dubiner, Spectral methods on triangles and other domains, J. Sci. Comp. 6 (1991), 345-390.

77. K. Eriksson and C. Johnson, Error estimates and automatic time step control for nonlinear parabolic problems, SIAM J. Numer. Anal. 24 (1987), 12-23.

78. _ Adaptive finite element methods for parabolic problems I: A linear model problem, SIAM J. Numer. Anal. 28 (1991), 43-77.

79. _ Adaptive finite element methods for parabolic problems II: Optimal error estimates in $l_{\infty} l_{2}$ and $l_{\infty} l_{\infty}$, SIAM J. Numer. Anal. 32 (1995), 706-740.

80. _ Adaptive finite element methods for parabolic problems IV: A nonlinear model problem, SIAM J. Numer. Anal. 32 (1995), 1729-1749.

81. _ Adaptive finite element methods for parabolic problems V: Long time integration, SIAM J. Numer. Anal. 32 (1995), 1750-1762.

82. K. Eriksson, C. Johnson, and V. Thomée, Time discretization of parabolic problems by the discontinuous Galerkin method, RAIRO, Anal. Numér. 19 (1985), 611-643.

83. D. Estep, A posteriori error bounds and global error control for approximation of ordinary differential equations, SIAM J. Numer. Anal. 32 (1995), 1-48.

84. D. Estep and D. French, Global error control for the continuous Galerkin finite element method for ordinary differential equations, RAIRO, Anal. Numér. 28 (1994), 815-852. 
85. D.J. Estep and R.W. Freund, Using Krylov-subspace iterations in discontinuous Galerkin methods for nonlinear reaction-diffusion systems, this volume, 1999, pp. -.

86. R. Falk, Analysis of finite element methods for linear hyperbolic problems, First International Symposium on Discontinuous Galerkin Methods (B. Cockburn, G.E. Karniadakis, and C.-W. Shu, eds.), Lecture Notes in Computational Science and Engineering, vol. 33, Springer Verlag, May 1999, pp.--.

87. R.S. Falk and G.R. Richter, Explicit finite element methods for symmetric hyperbolic equations, SIAM J. Numer. Anal., to appear.

88. J. Flaherty, R.M. Loy, M.S. Shephard, and J. Teresco, Software for the parallel adaptive solution of conservation laws by a discontinuous Galerkin method, this volume, 1999, pp. - .

89. J.E. Flaherty, R.M. Loy, C. Özturan, M.S. Shephard, B.K. Szymanski, J.D. Teresco, and L.H. Ziantz, Parallel structures and dynamic load balancing for adaptive finite element computation, Appl. Numer. Math. 26 (1998), 241-265.

90. J.E. Flaherty, R.M. Loy, M.S. Shephard, M.L. Simone, B.K. Szymanski, J.D. Teresco, and L.H. Ziantz, Distributed octree data structures and local refinement method for the parallel solution of three-dimensional conservation laws, Grid Generation and Adaptive Algorithms (Minneapolis) (M.W. Bern, J.E. Flaherty, and M. Luskin, eds.), The IMA Volumes in Mathematics and its Applications, vol. 113, Institute for Mathematics and its Applications, Springer, 1999, pp. 113-134.

91. J.E. Flaherty, R.M. Loy, M.S. Shephard, B.K. Szymanski, J.D. Teresco, and L.H. Ziantz, Adaptive local refinement with octree load-balancing for the parallel solution of three-dimensional conservation laws, J. Parallel and Dist. Comput. 47 (1997), 139-152.

92. A. Fortin, A. Béliveau, M.C. Heuzey, and A. Lioret, Ten years using discontinuous Galerkin methods for polymer processing problems, this volume, 1999, pp. -.

93. M. Fortin and A. Fortin, New approach for the finite element method simulation of viscoelastic flows, J. Non-Newt. Fluid Mech. 32 (1989), 295-310.

94. GAMM Workshop, December 4-6 1985, Nice, France, Numerical simulation of compressible Navier-Stokes equations - external 2D flows around a NACA0012 airfoil, Ed. INRIA, Centre de Rocquefort, de Rennes et de Sophia-Antipolis, 1986.

95. I.G. Giannakouros, Spectral element/Flux-Corrected methods for unsteady compressible viscous flows, Ph.D. thesis, Princeton University, Dept. of Mechanical and Aerospace Engineering, 1994.

96. J. Goodman and R. LeVeque, On the accuracy of stable schemes for $2 D$ scalar conservation laws, Math. Comp. 45 (1985), 15-21.

97. J. Greenstadt, An abridged history of cell discretization, this volume, 1999, pp. - .

98. P.-A. Gremaud, Simulation of granular flows, this volume, 1999, pp. -.

99. D.W. Halt, A compact higher order Euler solver for unstructured grids, Ph.D. thesis, Washington University, 1992.

100. D.W. Halt and R.K. Agarwall, A compact higher order characteristic-based Euler solver for unstructured grids, September, 1991, AIAA 91-3234.

101. A A compact higher order Euler solver for unstructured grids with curved boundaries, June, 1992, AIAA 92-2696. 
102. P. Houston, C. Schwab, and E. Süli, Stabilized hp-finite element methods for hyperbolic problems, SIAM J. Numer. Anal., to appear.

103. C. Hu, O. Lepsky, and C.-W. Shu, The effect of the lest square procedure for discontinuous Galerkin methods for Hamilton-Jacobi equations, this volume, 1999, pp. -

104. C. Hu and C.-W. Shu, A discontinuous Galerkin finite element method for Hamilton-Jacobi equations, SIAM J. Sci. Comput., to appear.

105. T. Hughes, G. Engel, L. Mazzei, and M. Larson, A comparison of discontinuous and continuous Galerkin methods, this volume, 1999, pp. -.

106. Hulbert and Hughes, Space-time finite element methods for second-order hyperbolic equations, Comput. Methods Appl. Mech. Engrg. 84 (1990), 327-348.

107. B. L. Hulme, One-step piecewise polynomial Galerkin methods for initial value problems, Math. Comp. 26 (1972), 415-426.

108. One-step piecewise polynomial Galerkin methods for initial value problems, Math. Comp. 26 (1972), 881-891.

109. J. Jaffré, C. Johnson, and A. Szepessy, Convergence of the discontinuous Galerkin finite element method for hyperbolic conservation laws, Mathematical Models \& Methods in Applied Sciences 5 (1995), 367-386.

110. P. Jamet, Galerkin-type approximations which are discontinuous in time for parabolic equations in a variable domain, SIAM J. Numer. Anal. 15 (1978), 912-928.

111. G. Jiang and C.-W. Shu, On cell entropy inequality for discontinuous Galerkin methods, Math. Comp. 62 (1994), 531-538.

112. C. Johnson, Error estimates and adaptive time-step control for a class of onestep methods for stiff ordinary differential equations, SIAM J. Numer. Anal. 25 (1988), 908-926.

113. C. Johnson and J. Pitkäranta, An analysis of the discontinuous Galerkin method for a scalar hyperbolic equation, Math. Comp. 46 (1986), 1-26.

114. G.E. Karniadakis and S.J. Sherwin, Spectral/hp element methods in CFD, Oxford University Press, 1999.

115. G. Karypis and V. Kumar, METIS Unstructured graph partitioning and sparse matrix ordering system version 2.0, Dept. of Computer Science, University of Minnesota, 1995.

116. D.A. Kopriva, S.L. Woodruff, and M.Y. Hussaini, Discontinuous spectral element approximation of Maxwell's equations, this volume, 1999, pp. -.

117. P. LeSaint and P.A. Raviart, On a finite element method for solving the neutron transport equation, Mathematical aspects of finite elements in partial differential equations (C. de Boor, ed.), Academic Press, 1974, pp. 89-145.

118. Q. Lin, Full convergence for hyperbolic finite elements, this volume, 1999, pp.--

119. Q. Lin, N. Yan, and A.-H. Zhou, An optimal error estimate of the discontinuous Galerkin method, Journal of Engineering Mathematics 13 (1996), 101-105.

120. Q. Lin and A. Zhou, A rectangle test for the first order hyperbolic equation, Proc. Sys. Sci. \& Sys., Great Wall Culture Publ. Co., Hong Kong (1991), 234-235.

121. Q. Lin and A.-H. Zhou, Convergence of the discontinuous Galerkin method for a scalar hyperbolic equation, Acta Math. Sci. 13 (1993), 207-210.

122. J.-G. Liu and C.-W. Shu, Numerical results on a high-order discontinuosu Galerkin method for $2 D$ incompressible flows, this volume, 1999, pp. -.

123. I. Lomtev and G.E. Karniadakis, A discontinuous Galerkin method for the Navier-Stokes equations, Int. J. Numer. Meth. Fluids 29 (1999), 587-603. 
124. I. Lomtev, R.M. Kirby, and G.E. Karniadakis, $A$ discontinuous Galerkin $A L E$ method for compressible viscous flow in moving domains, this volume, 1999, pp. - .

125. I. Lomtev, C.W. Quillen, and G.E. Karniadakis, Spectral/hp methods for viscous compressible flows on unstructured 2D meshes, J. Comput. Phys. 144 (1998), 325-357.

126. R. B. Lowrie, P. L. Roe, and B. van Leer, A space-time discontinuous Galerkin method for the time-accurate numerical solution of hyperbolic conservation laws, Proceedings of the 12th AIAA Computational Fluid Dynamics Conference, 1995, Paper 95-1658.

127. L Space-time methods for hyperbolic conservation laws, Barriers and Challenges in Computational Fluid Dynamics, ICASE/LaRC Interdisciplinary Series in Science and Engineering, vol. 6, Kluwer, 1998, pp. 79-98.

128. R.B. Lowrie, Compact higher-order numerical methods for hyperbolic conservation laws, Ph.D. thesis, University of Michigan, 1996.

129. R.B. Lowrie and J. Morel, Discontinuous Galerkin for hyperbolic systems with stiff relaxation, this volume, 1999, pp. - .

130. L. Machiels, A posteriori finite element output bounds of discontinuous Galerkin discretizations of parabolic problems, this volume, 1999, pp. -.

131. Y. Maday and E.M. Ronquist, Optimal error analysis of spectral methods with emphasis on non-constant coefficients and deformed geometries, Spectral and high order methods for partial differential equations (Como, Italy, 1989) (C. Canuto and A. Quarteroni, eds.), North-Holland, 1990, pp. 91-115.

132. X. Makridakis and I. Babuška, On the stability of the discontinuous Galerkin method for the heat equation, SIAM J. Numer. Anal. 34 (1997), 389-401.

133. J.T. Oden, Ivo Babuška, and C.E. Baumann, A discontinuous hp finite element method for diffusion problems, J. Comput. Phys. 146 (1998), 491-519.

134. J.T. Oden and L.C. Wellford, Jr., Discontinuous finite element approximations for the analysis of acceleration waves in eslastic solids, The Mathematics of finite element methods and applications II (J.R. Whiteman, Ed.) Academic Press, London (1976), 269-284.

135. T.J. Oden and C.E. Baumann, A conservative discontinuous Galerkin method for convection-diffusion and Navier-Stokes problems, this volume, 1999, pp.--.

136. S.A. Orszag and C. Tang, Small-scale structure of two-dimensional magnetohydrodynamic turbulence, J. Fluid Mech. 90 (1979), no. 1, 129-143.

137. S. Osher and J. Sethian, Fronts propagating with curvature dependent speed: Algorithms based on Hamilton-Jacobi formulations, J. Comput. Phys. 79 (1988), 12-49.

138. C. Ozturan, H.L. deCougny, M.S. Shephard, and J.E. Flaherty, Parallel adaptive mesh refinement and redistribution on distributed memory computers, Comput. Methods Appl. Mech. Engrg. 119 (1994), 123-137.

139. J. Peiro, J. Peraire, and K. Morgan, Felisa system- reference manual, Dept. of Aeronautics, Imperial College, 1994.

140. T. Peterson, A note on the convergence of the discontinuous Galerkin method for a scalar hyperbolic equation, SIAM J. Numer. Anal. 28 (1991), 133-140.

141. K.G. Powell, An approximate Riemann solver for magnetohydrodynamics (that works in more than one dimension), Tech. Report ICASE Report 94-24, ICASE, NASA Langley, 1994. 
142. M.K. Prasad, J.L. Milovich, A.I. Shestakov, D.S. Kershaw, and J.J. Shaw, $3 D$ unstructures mesh ALE hydrodynamics with the upwind discontinuous Galerkin method, this volume, 1999, pp. --

143. P. Rasetarinera, M.Y. Hussaini, and F.Q. Hu, Recent results in wave propagation analysis of the discontinuous Galerkin method, this volume, 1999, pp.--.

144. S. Rebay, GMRES for discontinuous Galerkin solution of the compressible Navier-Stokes equations, this volume, 1999, pp. - .

145. W.H. Reed and T.R. Hill, Triangular mesh methods for the neutron transport equation, Tech. Report LA-UR-73-479, Los Alamos Scientific Laboratory, 1973.

146. G.R. Richter, An optimal-order error estimate for the discontinuous Galerkin method, Math. Comp. 50 (1988), 75-88.

147. (1992), 631-643.

148. _ An explicit finite element method for the wave equation, Applied Numerical Mathematics 16 (1994), 65-80.

149. _ Explicit finite element methods for linear hyperbolic systems, this volume, 1999, pp. - .

150. B. Rivière and M.F. Wheeler, A discontinuous Galerkin method applied to nonlinear parabolic equations, this volume, 1999, pp. -.

151. Ch. Schwab, $p$ - and hp-finite element methods: Theory and applications in solid and fluid mechanics, Oxford University Press, Oxford, 1998.

152. _,$h p-F E M$ for fluid flow simulation, High-Order Methods for Computational Physics (T. Barth and H. Deconink, eds.), Lecture Notes in Computational Science and Engineering, vol. 9, Springer Verlag, 1999, pp. 325-438.

153. D. Schwanenberg and J. Kongeter, A discontinuous Galerkin method for the shalow water equations with source terms, this volume, 1999, pp.-.

154. S.J. Sherwin, Numerical phase properties analysis of the continuous and discontinuous Galerkin methods, this volume, 1999, pp.-.

155. C.-W. Shu and S. Osher, Efficient implementation of essentially nonoscillatory shock-capturing schemes, J. Comput. Phys. 77 (1988), 439-471.

156. __ Efficient implementation of essentially non-oscillatory shock capturing schemes, II, J. Comput. Phys. 83 (1989), 32-78.

157. N. Sobh, J. Huang, L. Yin, R.B. Haber, and D.A. Tortorelli, A discontinuous Galerkin model for precipitate nucleation and growth in aluminum alloy quench processes, Internat. J. Numer. Methods Engrg., to appear.

158. T. Strouboulis and J.T. Oden, A posteriori error estimation of finite element approximations in fluid mechanics, Comput. Methods Appl. Mech. Engrg. 78 (1990), 201-242.

159. E. Süli, A posteriori error analysis and global error control for adaptive finite element approximations of hyperbolic problems, Numerical Analysis 1995 (D.F. Griffiths and G.A. Watson, eds.), Pitman Lecture Notes in Mathematics Series, vol. 344, 1996, pp. 196-190.

160. A posteriori error analysis and adaptivity for finite element approximations of hyperbolic problems, An introduction to recent developments in theory and numerics for conservation laws (D. Kröner, M. Ohlberger, and C. Rhode, eds.), Lecture Notes in Computational Sciences and Engineering, vol. 5, Springer, 1999, pp. 123-194. 
161. E. Süli and P. Houston, Finite element methods for hyperbolic problems: A posteriori error analysis and adaptivity, The State of the Art in Numerical Analysis (I.S. Duff and G.A. Watson, eds.), Clarendon Press, Oxford, 1997, pp. 441-471.

162. E. Süli, Ch. Schwab, and P. Houston, hp-DGFEM for partial differential equations with non-negative characteristic form, this volume, 1999, pp. -.

163. J. Sun, M.D. Smith, R.C. Armstrong, and R. Brown, Finite element method for viscoelastic flows based on the discrete adaptive viscoelastic stress splitting and the discontinuous Galerkin method, Tech. report, Dept. Chemical Engineering, MIT, 1998.

164. H. Swann, The cell discretization algorithm: An overview, this volume, 1999, pp. -.

165. J.D. Teresco, M.W. Beall, J.E. Flaherty, and M.S.Shephard, A hierarchical partition model for adaptive finite element computation, Comput. Methods Appl. Mech. Engrg., submitted.

166. E. Toro, Riemann solvers and numerical methods for fluid dynamics, Springer Verlag, 1997.

167. H. van der Ven and J.J.W. van der Vegt, Accuracy, resolution, and computational complexity of a discontinuous Galerkin finite element method, this volume, 1999, pp. - .

168. B. van Leer, Towards the ultimate conservation difference scheme, II, J. Comput. Phys. 14 (1974), 361-376.

169. T. Warburton, Application of the discontinuous Galerkin method to Maxwell's equations using unstructured polymorphic hp-finite elements, this volume, 1999, pp. -.

170. T.C. Warburton, Spectral/hp methods on polymorphic multi-domains: Algorithms and applications, Ph.D. thesis, Brown University, 1998.

171. T.C. Warburton and G.E. Karniadakis, A discontinuous Galerkin method for the viscous MHD equations, J. Comput. Phys. 152 (1999), 1-34.

172. T.C. Warburton, I. Lomtev, R.M. Kirby, and G.E. Karniadakis, A discontinuous Galerkin method for the Navier-Stokes equations in hybrid grids, 10th. International Conference on Finite Elements in Fluids, Tucson, Arizona (M. Hafez and J.C. Heinrich, eds.), 1998.

173. L.C. Wellford, Jr. and J.T. Oden, A theory of discontinuous finite element approximations for the analysis of shock waves in nonlinear elastic materials, J. Comput. Phys. 19 (1975), 179-210.

174. A A theory of discontinuous finite element approximations for of shock waves in nonlinear elastic solids: Variational theory, Comput. Methods Appl. Mech. Engrg. 8 (1976), 1-16.

175. _ A theory of discontinuous finite element approximations for of shock waves in nonlinear elastic solids: Accuracy and convergence, Comput. Methods Appl. Mech. Engrg. 8 (1976), 17-36.

176. M.F. Wheeler, An elliptic collocation-finite element method with interior penalties, SIAM J. Numer. Anal. 15 (1978), 152-161.

177. M. Wierse, A new theoretically motivated higher order upwind scheme on unstructured grids of simplices, Adv. Comput. Math. 7 (1997), 303-335.

178. P. Woodward and P. Colella, The numerical simulation of two-dimensional fluid flow with strong shocks, J. Comput. Phys. 54 (1984), 115-173. 
179. L. Yin, A. Acharya, N. Sobh, R.B.Haber, and D.A.Tortorelli, A space-time discontinuous Galerkin method for elastodynamic analysis, this volume, 1999, pp. - .

180. A. Zhou and Q. Lin, Optimal and superconvergence estimates of the finite element method for a scalar hyperbolic equation, Acta Math. Sci. 14 (1994), $90-94$. 